\title{
Reduction of Spike Afterdepolarization by Increased Leak Conductance Alters Interspike Interval Variability
}

\author{
Fernando R. Fernandez and John A. White \\ Department of Bioengineering, University of Utah, Salt Lake City, Utah 84112
}

\begin{abstract}
Data from neurons in vivo have shown that spike output can often sustain episodes of high variability. Theoretical studies have indicated that the high conductance state of neurons brought on by synaptic activity can contribute to an increase in the variability of spike output by decreasing the integration timescale of the neuron. In the present work, we were interested in understanding how background synaptic conductance activity alters the interspike interval (ISI) variability of layer III pyramidal cells of the medial entorhinal cortex. We compared ISI variability in pyramidal cells as a result of synaptic current- or conductance-mediated membrane fluctuations. We found that the effects of background synaptic conductance activity on ISI variability depend on the neuron type. In pyramidal cells lacking spike frequency adaptation, the variability increased in relation to a comparable synaptic current stimulus. In contrast, in pyramidal cells displaying spike frequency adaptation, the synaptic conductance stimulus produced lower ISI variability. To understand this result, we constructed a phenomenological model that reproduced the basic properties of these neurons under control and increased leak conductance. We found that leak can change the properties of the neuron by acting as a bifurcation parameter that reduces the afterdepolarization (ADP) and decreases the slope (gain) of the frequency-current relationship, particularly for transient stimuli. A lower gain with the added leak causes a reduction in ISI variability. We conclude that the ability of a high conductance state to increase ISI variability cannot be generalized and can depend on the spike ADP dynamics expressed by the neuron.
\end{abstract}

Key words: membrane conductance; medial entorhinal cortex; layer III; CV; dynamic clamp; bifurcation; gain; afterdepolarization; ADP; afterhyperpolarization; AHP

\section{Introduction}

Recordings of neurons in vivo have shown that neuronal output is often characterized by a high degree of variability in interspike interval (ISI) times (Softky and Koch, 1993; Shadlen and Newsome, 1998; Anderson et al., 2000). Synaptic activity has also been shown to significantly increase the membrane conductance in vivo (Borg-Graham et al., 1998; Steriade et al., 2001; Mariño et al., 2005; Jorntell and Ekerot, 2006; Berg et al., 2007; Rudolph et al., 2007). Theoretical work has linked these two findings, showing that a high conductance state can contribute to an increase in ISI variability by reducing the time constant of the neuron (Stein, 1965; Bernander et al., 1991; Koch, 1999; Moreno-Bote and Parga, 2005).

Noise in synaptic activity can also alter neuronal spike output. In particular, the interaction between the threshold nonlinearity and noise has been exploited to smooth the output functions of neurons (Anderson et al., 2000; Longtin, 2000; Chance et al., 2002; Miller and Troyer, 2002; Wolfart et al., 2005). The ability for the steady-state leak conductance associated with background

Received Sept. 3, 2008; revised Nov. 7, 2008; accepted Dec. 10, 2008.

This work was supported by grants from the Canadian Institutes of Health Research (F.R.F.) and the National Institutes of Health (J.A.W.). We thank W. Hamish Mehaffey, Tilman J. Kispersky, and Michael N. Economo for useful discussions and comments on this manuscript.

Correspondence should be addressed to Fernando R. Fernandez, Department of Bioengineering, University of Utah, 20 South 2030 East 108, Biopolymers Research Building, Salt Lake City, UT 84112. E-mail: f.fernandez@utah.edu.

D01:10.1523/JNEUROSCI.4195-08.2009

Copyright $\odot 2009$ Society for Neuroscience $\quad$ 0270-6474/09/290973-14\$15.00/0 synaptic activity to change neuronal dynamics, outside of the expected reduction in time constant, is less well established. Recent studies, however, have shown that conservative increases in leak conductance can alter intrinsic cell dynamics (Prescott et al., 2006; Fernandez and White, 2008). In particular, a study of layer II stellate cells in the medial entorhinal cortex (MEC) has shown that increased leak conductance can reduce subthreshold membrane resonance and alter the power spectrum of the spike train (Fernandez and White, 2008). The ability for changes in leak conductance to influence ISI variability by altering neuronal firing dynamics, however, has not been considered. The increase in leak conductance could, potentially, create more complex relationships between conductance and ISI variability than those expected from the theoretical work.

To address the issue of how conductance inputs alter ISI variability, we used dynamic clamp to introduce artificial synaptic activity to layer III pyramidal cells in the MEC. Previous work in the MEC has emphasized the ability for intrinsic neuronal properties to pace spike output and influence network behavior within the hippocampal formation (Alonso and Llinás, 1989; Egorov et al., 2002; Giocomo et al., 2007). Consequently, it is important to understand how background synaptic conductance activity influences the regularity of spike output. To this end, we compared the ability of conductance-based inputs to alter ISI variability in two different types of layer III pyramidal neurons, discernable on the basis of spike frequency adaptation, the magnitude of the afterdepolarization (ADP), and membrane time constant. We 
demonstrate that the capacity of a high conductance state to influence the variability of the ISI distribution can depend on the ability for changes in conductance to modify the ADP after a spike and the gain of the frequency-current relationship. By reducing the ADP and gain, increased leak conductance can reduce ISI variability. Our results demonstrate that the effects of leak on ISI variability can be more complex than predicated in past theoretical studies.

\section{Materials and Methods}

Tissue preparation. All experimental protocols were approved by the University of Utah Institutional Animal Care and Use Committee. Horizontal sections of hippocampus and entorhinal cortex were prepared from 21- to 31-d-old Long-Evans rats. All chemicals were obtained from Sigma unless otherwise noted. After the animals were anesthetized with isoflurane and killed, brains were removed and immersed in $0^{\circ} \mathrm{C}$ artificial CSF (ACSF) consisting of the following (in $\mathrm{mm}$ ): $125 \mathrm{NaCl}, 25 \mathrm{NaHCO}_{3}$, 25 D-glucose, $2 \mathrm{KCl}, 2 \mathrm{CaCl}_{2}, 1.25 \mathrm{NaH}_{2} \mathrm{PO}_{4}$, and $1 \mathrm{MgCl}_{2}$ (buffered to pH 7.4 with $95 \% \mathrm{O}_{2} / 5 \% \mathrm{CO}_{2}$ ). Horizontal slices were cut to a thickness of $400 \mu \mathrm{m}$ (Vibratome $1000+$; Vibratome). Slices were incubated in a $32^{\circ} \mathrm{C}$ bubbled ACSF for $30 \mathrm{~min}$ before being cooled to room temperature $\left(20^{\circ} \mathrm{C}\right)$. After the incubation period, slices were moved to the stage of an infrared differential interference contrast-equipped microscope (Axioscope 2+; Zeiss). Slices were bathed in ACSF with $10 \mu \mathrm{M}$ CNQX, $50 \mu \mathrm{M}$ picrotoxin, and $30 \mu \mathrm{M} \mathrm{AP}-5$ to block ionotropic synaptic activity. All recordings were conducted between 32 and $34^{\circ} \mathrm{C}$.

Electrophysiology. Electrodes were drawn on a horizontal puller (P97; Sutter Instruments) and filled with an intracellular solution consisting of the following (in $\mathrm{mm}$ ): $120 \mathrm{~K}$-gluconate, $20 \mathrm{KCl}, 10 \mathrm{HEPES}, 7$ diTrisPhCr, $4 \mathrm{Na}_{2} \mathrm{ATP}, 2 \mathrm{MgCl}_{2}, 0.3$ Tris-GTP, and 0.2 EGTA, buffered to $\mathrm{pH} 7.3$ with $\mathrm{KOH}$. Final electrode resistances were between 3 and $4 \mathrm{M} \Omega$, with access values between 4 and $12 \mathrm{M} \Omega$. Electrophysiological recordings were performed with a current-clamp amplifier (Axoclamp 2A; Molecular Devices), and data were acquired using custom software developed in Matlab (version 2007b; MathWorks) using the data acquisition toolbox.

For dynamic-clamp experiments, the current-clamp amplifier was driven by an analog signal from an $86 \times$ personal computer running Real-Time Application Interface Linux and an updated version of the Real-Time Linux Dynamic Clamp (Dorval et al., 2001) called Real-Time Experimental Interface (Bettencourt et al., 2008). For all experiments, the sample rate of the dynamic clamp was set between 5 and $10 \mathrm{kHz}$. A measured junction potential of $\sim 10 \mathrm{mV}$ was subtracted from all recordings and taken into account during dynamic-clamp experiments. Data were collected at $2.5 \mathrm{kHz}$ and filtered at $1 \mathrm{kHz}$. Gain measurements of the frequency-current $(F-I)$ were made between frequencies above $0 \mathrm{~Hz}$ and the last frequency value within the linear range of the $F-I$ relationship (usually below $15 \mathrm{~Hz}$ ). For spike trains, the coefficient of variability (CV) was measured as the SD of the interspike interval (ISI) distribution divided by the mean of the distribution. Membrane resistance $\left(R_{i}\right)$ and time constant $\left(\tau_{m}\right)$ measurements were made in the linear region of the voltage-current relationship ( -90 to $-75 \mathrm{mV}$ ). Significance was determined using a paired Student's $t$ test. Means are presented with the SEM.

Protocols. Synaptic protocols consisted of two independent Poisson processes generating unitary synaptic events. Individual synaptic events were modeled using a biexponential function. For inhibition the rise and decay time constants were 0.5 and $5 \mathrm{~ms}$, respectively, whereas for excitation these values were 0.25 and $2.5 \mathrm{~ms}$. Inhibitory and excitatory events were delivered at a frequency of 1200 and $200 \mathrm{~Hz}$, respectively. The reversal value for excitation was set to $0 \mathrm{mV}$, whereas that for inhibition was $-75 \mathrm{mV}$. For excitation and inhibition, individual synaptic events had the same maximal conductance, which ranged from 0.5 to $0.6 \mathrm{nS}$. The ratio of inhibition to excitation is consistent with previous work in vitro that has measured spontaneous activity in layer III MEC (Greenhill and Jones, 2007). For current-based synaptic protocols, the reversal term was not included and maximal current for individual events was set between 10 and $13 \mathrm{pA}$. Note that individual maximal conductance or current for simulated synapses was slightly varied to produce the same fluctuation sizes (as determined by the SD of the membrane response) in different cells. Under conditions in which a steady-state leak conductance was used, we added $6 \mathrm{nS}$ of conductance with a reversal potential of either -70 or $-30 \mathrm{mV}$. Noise under conditions in which the leak was added as a steady-state conductance was implemented as a Gaussian current input with a cutoff at $100 \mathrm{~Hz}$. Under control and with added leak conductance, the current noise stimulus had an SD of $34.2 \pm 2$ and $49 \pm$ $2 \mathrm{pA}$, respectively, to achieve an SD in membrane voltage between 2 and $2.5 \mathrm{mV}$ near threshold (approximately $-70 \mathrm{mV}$ ).

For the experiments using dynamic clamp to increase the size of the afterhyperpolarization (AHP), we modeled the current using a voltagedependent gating and time constant variable expressed as $I$ $=g_{\max } x\left(V_{\text {membrane }}+90\right)$, with $x$ obeying $\frac{d x}{d t}=\frac{x_{\infty}-x}{\tau(V)}$,

with $x_{\infty}$ defined as $\frac{1}{1+e^{\left(\left(V-V_{\text {half }} /-k\right)\right.}}\left(V_{\text {half }}=-35, k=3\right)$, and $\tau(V)$ defined as $\frac{200}{e^{\left(V_{\text {membrane }}+50\right) / 2}}-e^{\left(V_{\text {membrane }}+6\right) /-50}+10$.

A maximum conductance of $5 \mathrm{nS}$ was used for $I_{\mathrm{AHP}+}$.

Simulations. All simulations were solved in either Matlab or XPPAUT version 5.86 (Ermentrout, 2002) using a fourth-order Runge-Kutta solver at a time step of $0.002 \mathrm{~ms}$. All phase-plane and bifurcation analyses were performed in XPPAUT version 5.86.

Equations for model were as follows: for membrane voltage, $C \frac{d V}{d t}$ $=I_{E}-g_{\mathrm{Na}^{+}} m_{\infty} h\left(V-E_{\mathrm{Na}^{+}}\right)-g_{\mathrm{Ca}^{2}+\mathcal{C}_{\infty}}\left(V-E_{\mathrm{Ca}^{2+}}\right)-g_{\mathrm{K}^{+}}(1-h)$ $\cdot\left(V-E_{\mathrm{K}^{+}}\right)-g_{\text {adapt }} n\left(V-E_{\mathrm{K}^{+}}\right)-g_{\text {leak }}\left(V-E_{\text {leak }}\right)$;

for $\mathrm{Na}^{+}$conductance activation, $m_{\infty}=\frac{1}{1+e^{\left(\left(V-V_{\text {hall }}\right) /-k\right)}}\left(V_{\text {half }}=\right.$ $-15, k=5)$;

for $\mathrm{Ca}^{2+}$ conductance activation, $c_{\infty}=\frac{1}{1+e^{\left(\left(V-V_{\text {half }}\right) /-k\right)}}\left(V_{\text {half }}=\right.$ $-28, k=6.5)$;

for $\mathrm{Na}^{+}$conductance inactivation, $\frac{d h}{d t}=\frac{h_{\infty}-h}{\tau_{h}(V)}, h_{\infty}$ $=\frac{1}{1+e^{\left(\left(V-V_{\text {half }} /-k\right)\right.}}\left(V_{\text {half }}=-16, k=-5\right) \tau_{h}(V)=\frac{0.81}{e^{(V+38) / 5}}$ $-e^{(V+52) /-3}+0.24$

and for slow, $\mathrm{Ca}^{2+}$ activated conductance $\mathrm{K}^{+}$current, $\frac{d n}{d t}$ $=\frac{n_{\infty}-n}{\tau_{n}}\left(\tau_{n}=70_{m s}\right) n_{\infty}=\frac{1}{1+e^{\left(\left(\left[\mathrm{Ca}^{2+}\right]-0.018\right) /-0.000025\right)}},\left[\mathrm{Ca}^{2+}\right]=$ - 0.12( $\left.g_{\mathrm{Ca}^{2+}}\left(V-E_{\mathrm{Ca}^{2+}}\right)\right)$.

Additional model parameters were as follows: $C=1.5 \mu \mathrm{F} / \mathrm{cm}^{2} ; g_{\mathrm{Na}^{+}}=20$ $\mathrm{mS} / \mathrm{cm}^{2} ; g_{\mathrm{Ca}^{2+}}=0.025 \mathrm{mS} / \mathrm{cm}^{2} ; g_{K^{+}}=3.65 \mathrm{mS} / \mathrm{cm}^{2} ; g_{\text {adapt }}=0.18 \mathrm{mS} /$ $\mathrm{cm}^{2} ; g_{\text {leak }}=0.02 \mathrm{mS} / \mathrm{cm}^{2}\left(0.047 \mathrm{mS} / \mathrm{cm}^{2}\right.$ for high conductance state); $E_{\mathrm{Na}^{+}}=45 \mathrm{mV} ; E_{K^{+}}=-95 \mathrm{mV} ; E_{\mathrm{Ca}^{2+}}=120 \mathrm{mV} ; E_{\text {leak }}=-70 \mathrm{mV} . I_{E}$ in the current balance equation refers to driving current.

The $\mathrm{Na}^{+}$and $\mathrm{Ca}^{2+}$ conductance activations were modeled using only the steady state. These conductances are assumed to equilibrate instantaneously with membrane voltage. Also, the activation of the fast $\mathrm{K}^{+}$ conductance was modeled as $1-h$. These reductions allow for the model to contain only three dynamic variables $(V, h, n)$. All time constants are in units of milliseconds. Noise in the model was implemented as a Gaussian current input with a cutoff at $100 \mathrm{~Hz}$. Under control and with added leak conductance, the noise stimulus had an SD of 0.13 and $0.22 \mu \mathrm{A} / \mathrm{cm}^{2}$, respectively, to achieve an SD in membrane voltage (measured at -80 $\mathrm{mV}$ ) of $2.5 \mathrm{mV}$ under each condition.

\section{Results}

We started by comparing neuronal spike output in layer III pyramidal cells of MEC in response to synaptic input consisting of either conductance- or current-based artificial synaptic stimuli delivered via dynamic clamp. We could distinguish layer III pyramidal cells from neighboring layer II stellate cells because of an absence of either spike clustering or subthreshold theta oscilla- 
$\mathrm{Ai}$

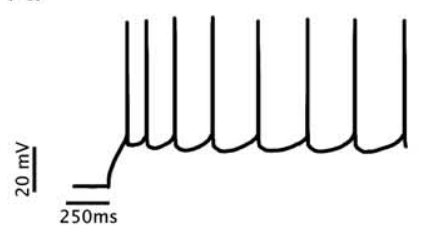

$\mathrm{Bi}$

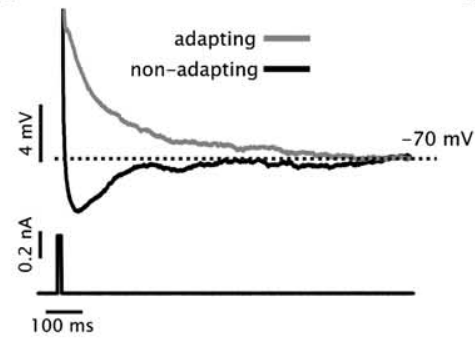

Adapting cell
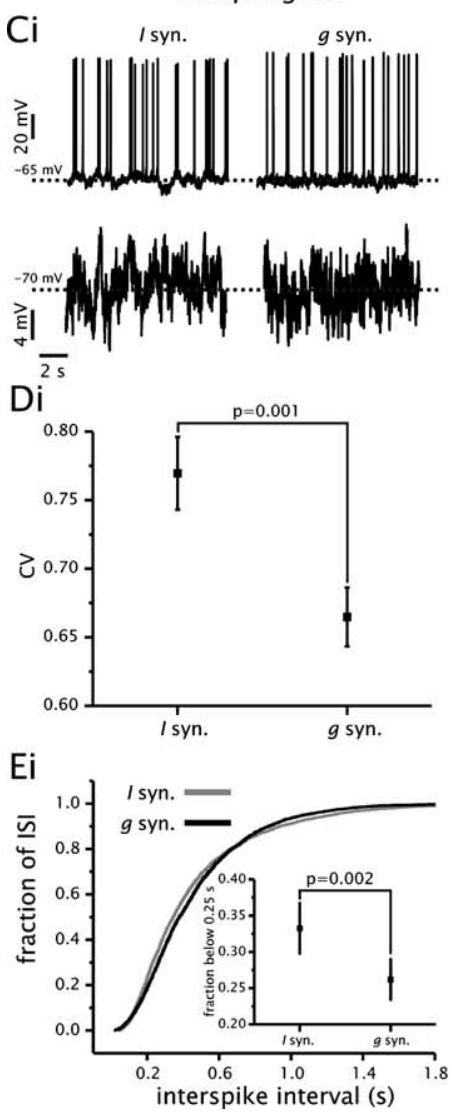

Aii

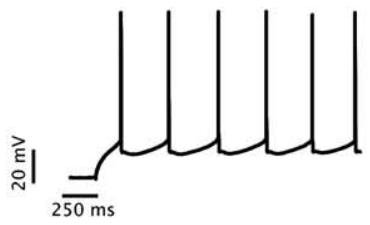

Bii

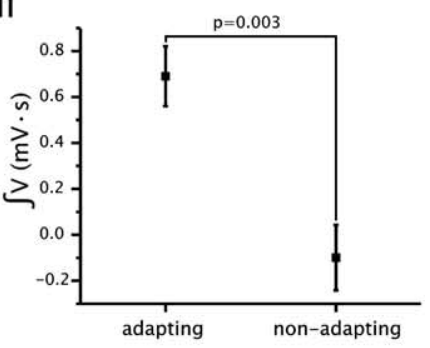

Non-adapting cell

Cii
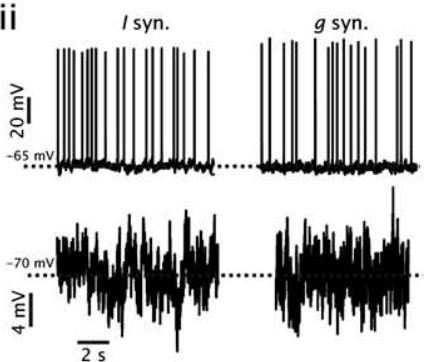

Dii

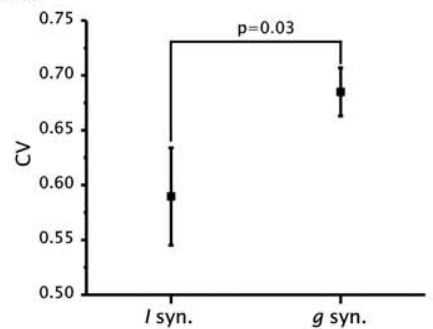

Eii

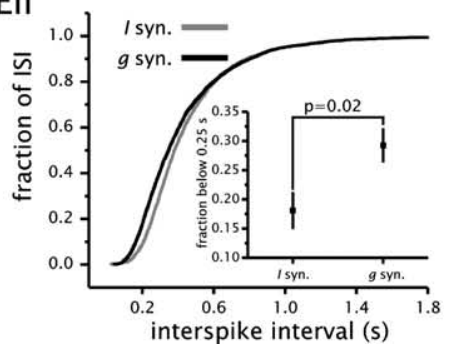

Figure 1. Adapting and non-adapting layer III pyramidal cells respond differently to conductance-based synaptic input. $\boldsymbol{A}$, Example of voltage traces elicited with a square current step from an adapting (Ai) and non-adapting (Aii) pyramidal cell. $\boldsymbol{B}$, Comparison of the ADP in adapting and non-adapting pyramidal cells generated during a pulse-elicited spike (Bi). The ADP was measured as the integral of the membrane voltage between the start and end ( $\sim 800 \mathrm{~ms}$ after the spike) of the ADP relative to the holding potential (Bii). C, Example of voltage traces from an adapting (Ci) and non-adapting (Cii) cell in the presence of a current-based (I syn.) or conductance-based ( $g$ syn.) stimulus in the subthreshold (bottom) or spiking (top) regime. D, Plots of the average $\mathrm{CV}$ in adapting (Di) and non-adapting (Dii) cells driven with a current- or conductance-based stimulus. $\boldsymbol{E}$, Cumulative distribution plots of ISIs from adapting (Ei) and non-adapting (Eii) cells driven with a current-based (gray) or conductance-based (black) stimulus. Cumulative distribution plots contain the aggregate of ISI values from all cells analyzed. For each panel, the insets show a plot of the average fraction of ISI values below $0.25 \mathrm{~s}$ for each stimulus condition (I syn. vs $g$ syn.).

tions (Klink and Alonso, 1993; Dickson et al., 1997; Erchova et al., 2004). Furthermore, layer III cells had a higher membrane input resistance $\left(R_{i}\right)$ than layer II cells (Dickson et al., 1997; Klink and Alonso, 1997).
Previous work has established the existence of heterogeneity in the electrophysiological properties of layer III pyramidal cells (Gloveli et al., 1997a,b). In support of this, we found that layer III pyramidal cells could be divided into two populations that could be distinguished on the basis of the expression of spike frequency adaptation (Fig. $1 A$ ) and differences in passive membrane properties. Under control conditions, adapting cells had an $R_{i}$ and time constant $\left(\tau_{m}\right)$ of $207 \pm 15 \mathrm{M} \Omega$ and $66 \pm 6$ $\mathrm{ms}(n=9)$, whereas non-adapting cells had values of $198 \pm 32 \mathrm{M} \Omega$ and $34 \pm 5 \mathrm{~ms}$ $(n=8)$, respectively. Note that only the $\tau_{m}$ differed significantly between the two cell subtypes $(p=0.002)$. Previous work has also shown that a long ADP is a distinguishing characteristic of layer III pyramidal cells (Yoshida and Alonso, 2007). Hence, we quantified the ADP in adapting and non-adapting cells. The size of ADP was quantified as the integral of the membrane voltage during the ADP. A $10 \mathrm{~ms}$ pulse of current ranging in amplitude from 0.4 to $0.6 \mathrm{nA}$ was used to elicit a single spike while holding cells at $-70 \mathrm{mV}$. As shown in Figure $1 B$, adapting cells produced a substantially larger ADP than non-adapting cells $(0.70 \pm 0.13$ vs $-0.1 \pm$ $0.14 \mathrm{mV} \cdot \mathrm{s} ; p=0.003 ; n=5)$. As a result of these data, we treated adapting and nonadapting cells within layer III as two separate and electrophysiologically discernable groups of neurons. This distinction allowed us to contrast the response of each cell type to the same conductance- and current-based stimuli.

Cell stimuli consisted of excitatory and inhibitory synapses driven at 200 and 1200 $\mathrm{Hz}$, respectively, by independent Poisson processes (see Materials and Methods). Parameter values for synaptic size and kinetics were chosen so as to generate subthreshold fluctuations with an SD between 2.0 and $2.5 \mathrm{mV}$ and a decrease in membrane resistance of $\sim 50 \%$ for the conductance stimuli $\left(R_{i}=101 \pm 17 \mathrm{M} \Omega\right.$ for adapting and $110 \pm 17 \mathrm{M} \Omega$ for nonadapting). The SD of the subthreshold membrane response for adapting cells was $2.2 \pm 0.12$ and $2.2 \pm 0.10 \mathrm{mV}$ under current- and conductance-based stimuli, respectively $(n=6)$. Similarly, for nonadapting cells, these values were $2.2 \pm 0.14$ and $2.0 \pm 0.12 \mathrm{mV}(n=6)$. These values are comparable with what has been recorded in vivo from other cortical regions (Destexhe et al., 2003; Cardin et al., 2008). Spike output variability under current- or conductance-based synaptic stimuli was quantified using the $\mathrm{CV}$ (the ratio of the $\mathrm{SD}$ to the mean) of the ISI distribution. Spike times were collected from $200 \mathrm{~s}$ sweeps for each condition (current and conductance 
stimuli; adapting and non-adapting cells) from cells firing $\sim 2$ $\mathrm{Hz}$. This value of firing frequency is consistent with in vivo singleunit recordings of excitatory cells from awake and behaving animals from the superficial layers of the MEC (Frank et al., 2001). Average firing frequency values for adapting cells were $2.2 \pm 0.12$ and $2.2 \pm 0.10 \mathrm{~Hz}(n=8)$, whereas mean values in non-adapting cells were $2.2 \pm 0.1$ and $2.3 \pm 0.13 \mathrm{~Hz}(n=8)$ under current- and conductance-based stimuli, respectively.

The ability for the conductance-based stimulus to increase the $\mathrm{CV}$ of the spike train above that of a comparable current-based stimulus depended on the type of layer III pyramidal cell. In adapting cells, the conductance stimulus decreased the CV from $0.77 \pm 0.03$ to $0.66 \pm 0.02(p=0.01 ; n=8)$ (Fig. $1 D i)$. However, in non-adapting cells, the conductance stimulus increased the CV from $0.59 \pm 0.04$ to $0.68 \pm 0.02(p=0.03 ; n=8)$ (Fig. 1 Dii $)$. The change in the $\mathrm{CV}$ of both cell types was caused by a corresponding change in the number of high-frequency spike events. To illustrate this result, we plotted the total cumulative distribution of ISIs for both types of cells under each stimulus condition (Fig. $1 E)$. In adapting cells, the cumulative distribution curve indicated a greater number of high-frequency events with a currentbased stimulus (Fig. $1 \mathrm{Ei}$ ). Conversely, in non-adapting cells, the cumulative distribution under the current-based stimulus contained fewer high-frequency events (Fig. 1 Eii). The conductance stimulus decreased the fraction of ISIs below $0.25 \mathrm{~s}(4 \mathrm{~Hz})$ from $0.33 \pm 0.03$ to $0.26 \pm 0.03$ in adapting cells $(p=0.002 ; n=8)$ and increased this fraction from $0.18 \pm 0.03$ to $0.29 \pm 0.03$ ( $p=$ $0.02 ; n=8$ ) in non-adapting cells (Fig. $1 E$, insets).

These results indicate that the ability of a high conductance state to increase ISI variability depends on the subtype of pyramidal cell. In non-adapting cells, the conductance-based stimulus increased the CV relative to a current-based stimulus. This is in agreement with previous theoretical work (Stein, 1965; Bernander et al., 1991; Koch, 1999) and can be explained by the shorter integration time under the higher conductance state. A leakier membrane leads to faster decay rates for synaptic inputs. As a result, more coincident activity is required to reach threshold as the neuron averages over fewer subthreshold inputs, which increases the variability of the spike output (Stein, 1965; Bernander et al., 1991; Koch, 1999). The ability of this mechanism to increase the $\mathrm{CV}$, however, cannot be generalized to adapting pyramidal cells, because the conductance stimulus produced a decrease in ISI variability. Although the synaptic conductance stimulus also reduced $R_{i}$ and $\tau_{m}$ in adapting cells, and hence also reduced the integration time of synaptic inputs, our data indicate that additional factors must be changing with the addition of leak conductance that are overcoming the potential increase in ISI variability. For the remainder of the study, we focused on the mechanisms controlling ISI variability in adapting pyramidal cells.

\section{Decrease in $\mathrm{CV}$ in adapting cells is caused by the increase in membrane conductance associated with synaptic activity}

To investigate the mechanism by which the synaptic conductance stimulus reduced the CV in adapting pyramidal cells, we started by introducing the increase in membrane conductance associated with the synaptic conductance stimulus separate from the noise component. The change in conductance was therefore introduced as a steady-state leak conductance. For the noise component of the stimulus, we used a current input consisting of a Gaussian noise source rather than synaptic waveforms. In contrast to our previous experiments using the synaptic conductance stimulus, this form of noise is additive because it is not multiplied by the membrane voltage term (i.e., driving force). Hence, we could test the effects of adding a leak conductance on cell firing dynamics without adding a stochastic component. This also allowed us to test whether our results with the synaptic conductance stimulus could be reproduced in a more general context independent of specific synaptic waveforms.

We recorded $200 \mathrm{~s}$ sweeps from cells firing at $\sim 2 \mathrm{~Hz}$ in the absence or presence of a steady-state leak conductance $(2.2 \pm$ $0.06 \mathrm{~Hz}$ in control and $2.1 \pm 0.07 \mathrm{~Hz}$ with added leak) along with a current-based Gaussian stimulus (see Materials and Methods). To match the change in $R_{i}$ associated with the synaptic conductance, we introduced a $6 \mathrm{nS}$ steady-state leak conductance via dynamic clamp $(207 \pm 15 \mathrm{M} \Omega$ in control and $100 \pm 3 \mathrm{M} \Omega$ with leak). As expected, the added leak conductance also reduced the membrane time constant within the subthreshold range (less than $-75 \mathrm{mV}$ ) of the membrane voltage from $66 \pm 6$ to $31.5 \pm$ $2.0 \mathrm{~ms}(p=0.0002 ; n=9)$ (see Fig. $3 D)$.

We initially set the reversal potential for the leak conductance at $-70 \mathrm{mV}$, which was equal to the total synaptic conductance stimulus used in the previous section. The magnitude of the Gaussian noise term was adjusted to maintain the same SD in subthreshold membrane voltage in control and leak modified cells $(2.3 \pm 0.16 \mathrm{mV}$ in control and $2.2 \pm 0.16 \mathrm{mV}$ with added leak).

As with the synaptic conductance stimulus, the presence of the added leak conductance reduced ISI variability relative to control (Fig. $2 B, C$ ). Figure $2 B$ shows four pairs of histograms of the ISI distribution from cells under control and with the added leak conductance. As indicated, the CV decreased from $0.70 \pm 0.03$ in control to $0.53 \pm 0.02$ with the added leak conductance $(n=17 ; p=3 \times$ $10^{-5}$ ) (Fig. 2C). The decrease in the CV with increased leak conductance was caused by a corresponding decrease in the number of high-frequency spike events (Fig. 2D). The fraction of ISI values below $0.25 \mathrm{~s}$ under control was $0.28 \pm 0.02$, whereas with the added leak conductance the value decreased to $0.16 \pm 0.02\left(p=2 \times 10^{-4}\right.$; $n=17$ ) (Fig. $2 D$, inset). We repeated these experiments with a more depolarized reversal potential $\left(E_{\mathrm{rev}}=-30 \mathrm{mV}\right)$ for the added leak conductance to test whether the decrease in ISI variability was dependent on the reversal potential. Because of the depolarizing nature of the leak, we used a negative direct current input to maintain cells at the same firing rate $(2.0 \pm 0.15 \mathrm{~Hz})$ and membrane potential as before. Under control, the CV was $0.70 \pm 0.03$, whereas in the presence of the depolarizing leak, the value decreased to $0.51 \pm 0.02(p=$ $\left.9 \times 10^{-4} ; n=7\right)$. Hence, the reversal potential for the leak conductance was not a determinant of whether increased conductance reduced ISI variability.

From the voltage traces shown in Figure $2 \mathrm{~A}$, it appeared that, under the spiking condition, the SD of the membrane voltage was greater in control than with the added leak conductance despite being the same in the subthreshold regime. To verify this, we compared the SD under control and with added leak in the spiking regime (Fig. $2 E$ ). In all cases, the membrane voltage distribution could be fit with a Gaussian function $\left(r^{2}>0.98\right)$ (Fig. $2 E$ ). A comparison of the SD during spiking confirmed that the value was significantly greater in control than with the added leak conductance ( $5.8 \pm 0.2$ vs $4.5 \pm 0.16 \mathrm{mV} ; p=0.01 ; n=5$ ) despite being the same in the subthreshold regime (Fig. $2 E$ ). This result suggested that the added leak conductance was uniquely altering the neuron in the spiking regime.

We were also interested in the effects of added leak conductance on the rate of spike frequency adaptation in the absence of a noisy stimulus, because previous work has shown that increasing leak can increase the rate of spike frequency adaptation (Prescott et al., 2006). Furthermore, a change in the rate of spike frequency adaptation could be a potential contributor to the changes observed in the ISI variability. To quantify the rate of 
Ai control

Aii
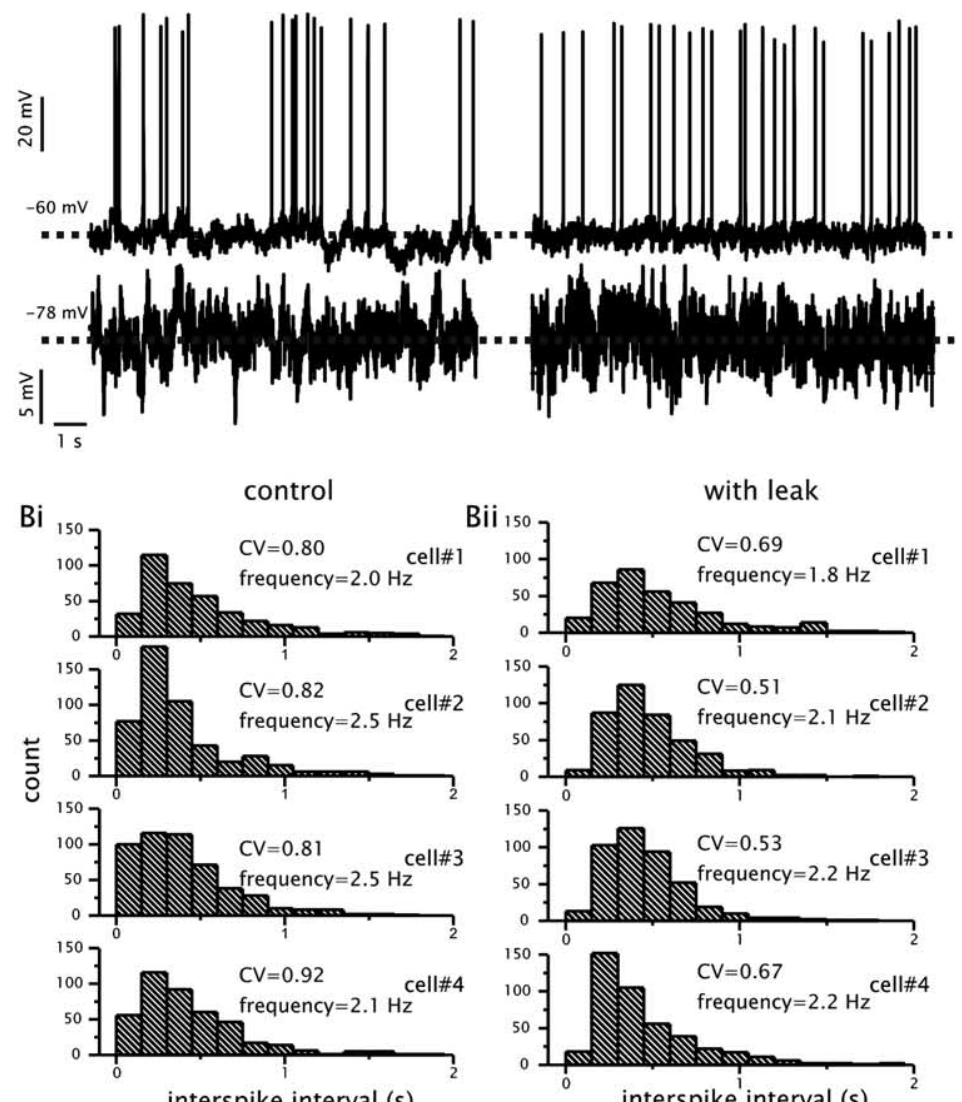

C

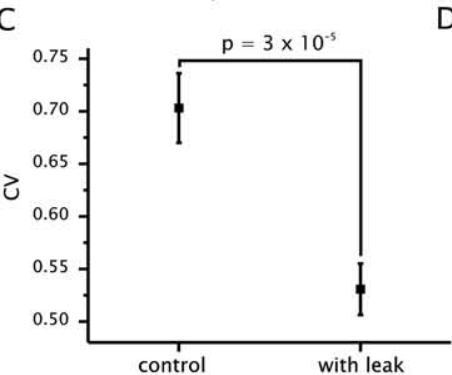

E

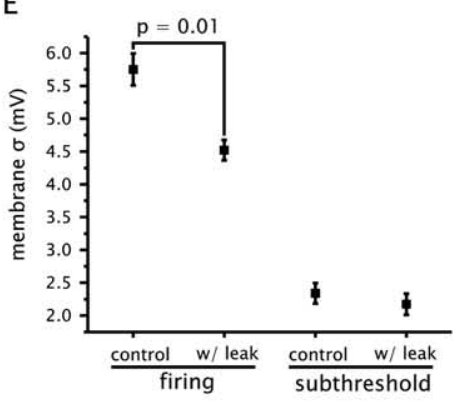

with leak

with leak
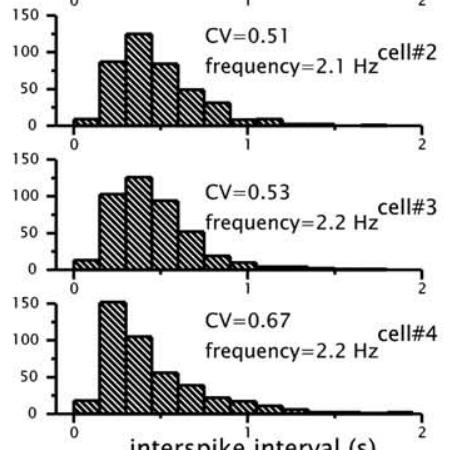

D
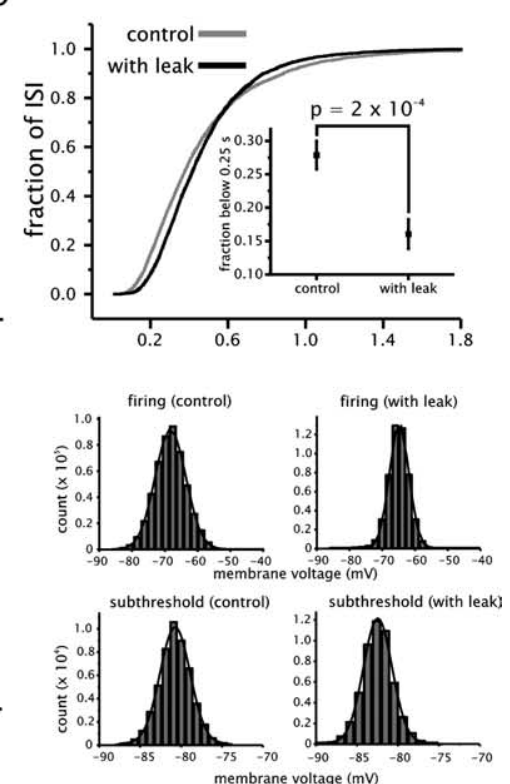

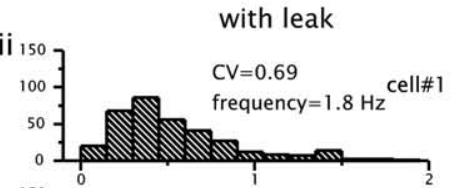

Figure 2. Adding an artificial steady-state leak conductance in adapting layer III pyramidal decreases ISI variability. $\boldsymbol{A}$, Example of voltage traces from an adapting pyramidal cell under control conditions (Ai) or with $6 \mathrm{nS}$ of added leak conductance (Aii) in the subthreshold (bottom) and spiking (top) regimes. Under both conditions, spiking was driven with a zero mean Gaussian current input (see Materials and Methods). $\boldsymbol{B}$, Examples of four pairs of ISI histograms from cells eliciting spikes in response to Gaussian current input in the absence (left column) or presence (right column) of an added leak conductance. Bin size for histograms was $0.15 \mathrm{~s}$. C, Plot of the average CV values in adapting cells driven in the absence or presence of an added leak conductance. $\boldsymbol{D}$, Cumulative distribution plots of the ISI values in the absence (gray) or presence (black) of added leak conductance. The inset shows a plot of the average fraction of ISI values below 0.25 s under each condition. $E$, Plot of the SD of membrane voltage in the spiking and subthreshold regimes with or without an added leak conductance (left). Right shows examples of histograms of membrane voltage distributions from a single cell in the subthreshold and spiking regime under control conditions or with added leak conductance. All distributions of membrane voltage could be fit with a Gaussian function.

adaptation, we provided cells with a step depolarization such that steady-state firing rate at the end of the current step was $\sim 5$ $\mathrm{Hz}$. We fit the firing frequency versus time relationship with a single-exponential function under control or with added leak conductance $\left(r^{2}=0.89 \pm 0.025 ; n=7\right)$ (Fig. $3 B$ ). Contrary to previous results in hippocampal pyramidal cells (Prescott et al., 2006), addition of the leak conductance increased the average time constant of adaptation from $0.11 \pm 0.03$ to $0.19 \pm$ $0.03 \mathrm{~s}(p=0.04 ; n=7)$ (Fig. $3 C)$.

In summary, adding a steady-state leak conductance to adapting layer III pyramidal cells increased spike output regularity, reduced the SD in membrane voltage during spiking, increased the time constant of adaptation, and reduced $\tau_{m}$.

Conductance-based model can reproduce decrease in $\mathrm{CV}$ induced by added leak conductance

In the previous section, we showed that the $\mathrm{SD}$ in membrane voltage was reduced significantly during spiking with the addition of leak compared with control. This result suggests that factors associated with the spike firing dynamics must be changing with the addition of leak conductance. As a result of changes associated with spike firing, an increase in leak conductance could result in a decrease in ISI variability. To begin to understand how increased leak conductance can decrease ISI variability, we started by building a conductance-based model that can account for the spike firing behavior in adapting pyramidal cells. Our goal was to build a model that could reproduce the effects of added leak conductance on ISI variability and spike frequency adaptation but simple enough that we could analyze using tools from nonlinear dynamics (e.g., phase-plane analysis, bifurcation theory). Using this approach, we can analyze the effects of adding leak conductance on the fundamental dynamics and output characteristics of the model.

The model incorporates four voltagegated conductances, which include the following: $\mathrm{Na}^{+}\left(I_{\mathrm{Na}}\right)$, fast $\mathrm{K}^{+}\left(I_{\mathrm{KF}}\right), \mathrm{Ca}^{2+}$ $\left(I_{\mathrm{Ca}}\right)$, and slow $\mathrm{Ca}^{2+}$ activated $\mathrm{K}^{+}\left(I_{\mathrm{KCa}}\right)$ currents. The model parameters were set to match the membrane time constant, adaptation rate, and membrane voltage range observed in experimental results under control conditions. To keep the model sufficiently simple for analysis, we used three differential equations to describe the following dynamic variables in the model: voltage, $I_{\mathrm{Na}}$ inactivation, and $I_{\mathrm{KCa}}$ activation. Hence, the model has three dimensions. Voltage and $I_{\mathrm{Na}}$ inactivation operate 
on a fast timescale (fast subsystem), whereas the variable controlling $I_{\mathrm{KCa}}$ activation $(n)$ is a slow variable (slow subsystem). We used an adaptation mechanism similar to previous models (Ermentrout, 1998). In essence, $I_{\mathrm{Ca}}$ determines the steady-state activation level of $I_{\mathrm{KCa}}$, which regulates the rate of adaptation in the firing frequency. This form of adaptation is consistent with previous experimental results showing a $\mathrm{Ca}^{2+}$-dependent adaptation mechanism in layer III pyramidal cells (Dickson et al., 1997). To incorporate the change in membrane conductance, we simply increased the maximal leak conductance of the model.

A significant constraint in constructing the model was reproducing the increase in the adaptation time constant of spike frequency with added leak conductance. This result was only observed under certain parameter regimes. Furthermore, we found that the increase in the time constant of spike frequency adaptation with added leak conductance could not account for the decrease observed in ISI variability. As a result, changing the activation time constant of the $n$ variable, so that the rate of spike frequency adaptation under control matched that with added leak conductance and vice versa, did not significantly change the ISI variability (supplemental Fig. 1, available at www.jneurosci.org as supplemental material). As a consequence, we considered the changes in adaptation as secondary and not principally involved in ISI variability but, nevertheless, an important observation for constraining the model. In the model, achieving the change in the spike frequency adaptation time constant and simulating the high conductance state required increasing the leak conductance from 0.02 to $0.047 \mathrm{mS} / \mathrm{cm}^{2}\left(\tau_{m}\right.$ was $75 \mathrm{~ms}$ in control vs $33 \mathrm{~ms}$ with extra leak). As shown in Figure 4, the model is able to reproduce the change in spike frequency adaptation with a time constant of $0.13 \mathrm{~s}$ in control and $0.18 \mathrm{~s}$ with the added leak conductance (Fig. 4A, $B$ ).

We proceeded to test whether the model could reproduce the effects of added leak conductance on ISI variability. We stimulated the model using Gaussian noise under control and an increased level of leak conductance $\left(0.047 \mathrm{mS} / \mathrm{cm}^{2}\right)$ for $200 \mathrm{~s}$ while maintaining an average firing frequency of $\sim 2.5 \mathrm{~Hz}$. We adjusted the magnitude of the noise input to maintain the SD of the subthreshold membrane voltage (measured at $-80 \mathrm{mV}$ ) at $2.5 \mathrm{mV}$ under both conditions. The model with control levels of leak produces a CV of 0.75 , whereas with increased leak conductance the $C V$ decreases to 0.60 (Fig. $4 C, D$ ). Similar to the experimental data, a plot of the cumulative ISI distribution shows a decrease in high-frequency events with added leak conductance (Fig. 4E).

As with the experimental data, we measured the SD of the membrane voltage during spiking under control and with the added leak conductance (Fig. $4 F$ ). During spiking, the SD rises from the set value of $2.5 \mathrm{mV}$ in the subthreshold regime (with Gaussian noise) to $5.8 \mathrm{mV}$ in control and $5.3 \mathrm{mV}$ with the added leak conductance. As with the experimental data, the membrane voltage distribution can be accurately fit with a Gaussian function added leak conductance.
$\mathrm{Bi}$

Bii

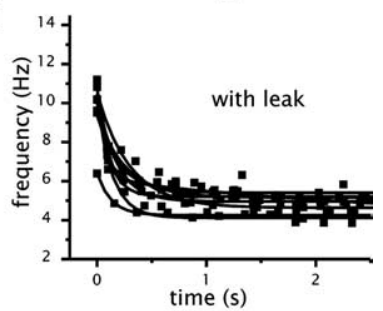

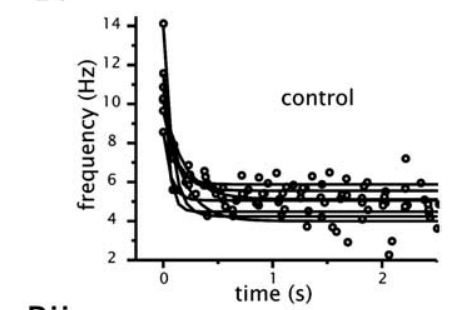

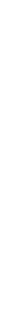

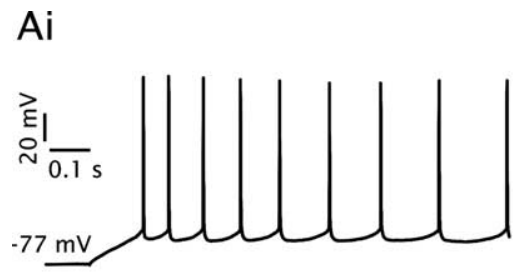

Figure 3. Adding a steady-state leak conductance in adapting pyramidal cells increases the rate of spike frequency adaptation. , with added leak conductance (Bii). Plots show data from seven cells under each condition. Lines denote a single-exponential fit to individual adaptation rates under each condition. C, Plot of average time constant of adaptation under control conditions and with

$\left(r^{2}>0.98\right)$ (Fig. 4F). Hence, the model reproduces the qualitative experimental results, although the decrease in the SD of the membrane voltage in the spiking regime with added leak conductance is more modest than in the experimental data.

\section{Increased leak conductance reduces the ADP of the fast subsystem in the pyramidal cell model}

To understand the dynamics in the model responsible for reducing ISI variability with increased leak conductance, we did a phase-plane and bifurcation analysis under control and increased leak conductance (for a review of these methods, see Izhikevich, 2007). The phase-plane analysis allows the nullclines, fixed points (e.g., stable membrane potential states), and voltage trajectories of the model to be simultaneously visualized. A bifurcation analysis allows the fixed points and limit cycles (e.g., spike firing solution) to be tracked as a model parameter (e.g., $I_{E}$ ) is changed. Together, these forms of analyses can provide insight into biophysical and dynamical issues. In particular, these analyses can track fundamental changes in the dynamics of a model and associate them with the output characteristics of the model (e.g., F-I relationship, phase response curve, oscillations), which can have an important impact on spike output regularity.

As indicated by our experimental and modeling data, the decrease in CV with added leak conductance was caused by a corresponding decrease in high-frequency spike events. To help understand this phenomenon, we considered the fast subsystem of the model. We froze the adaptation variable $(n)$ controlling $I_{\mathrm{KCa}}$ at a value achieved during high-frequency spike events but below the maximum value attained during the simulations with Gauss- 
Ai

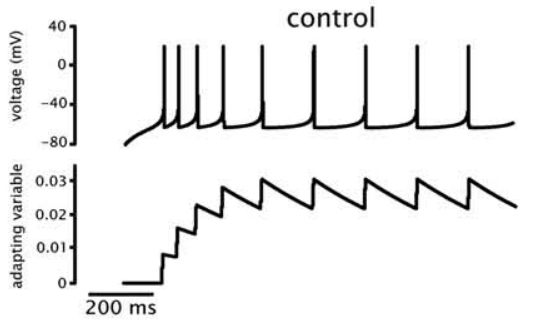

Aii

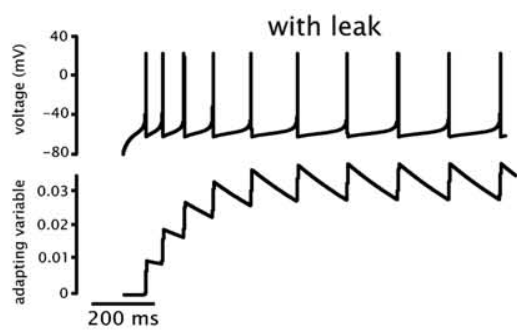

C
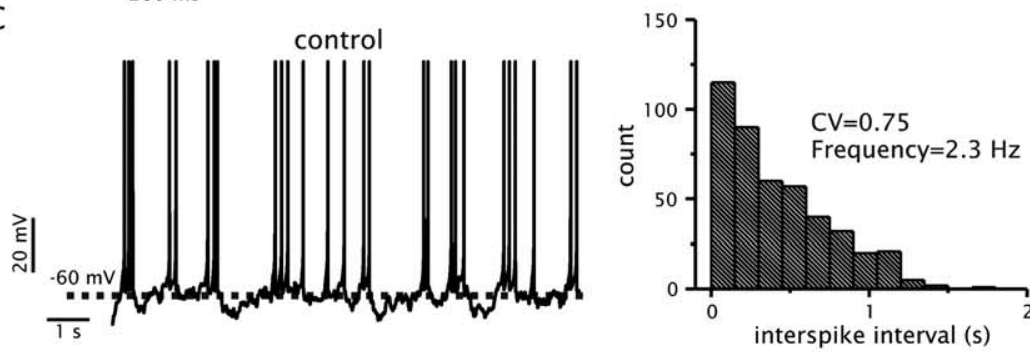

D
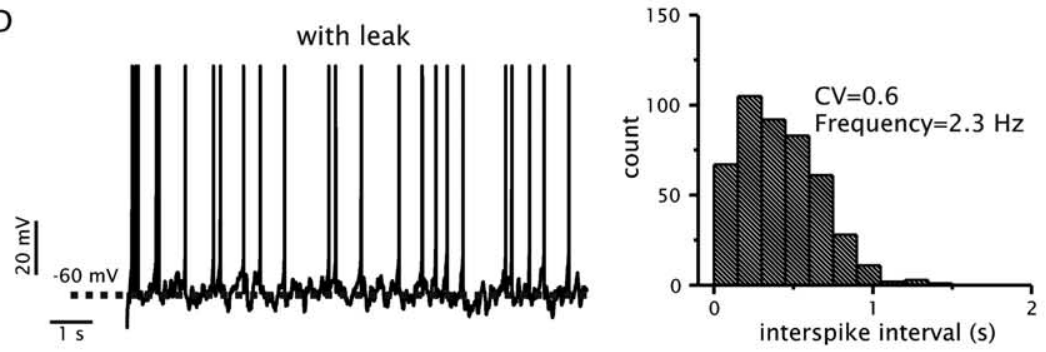

$E$

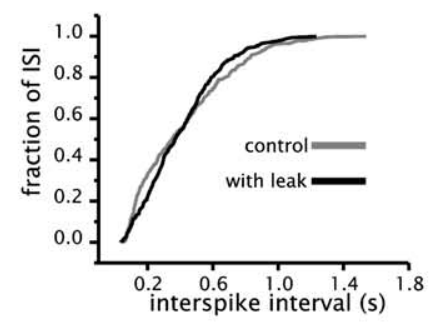

$\mathrm{F}$
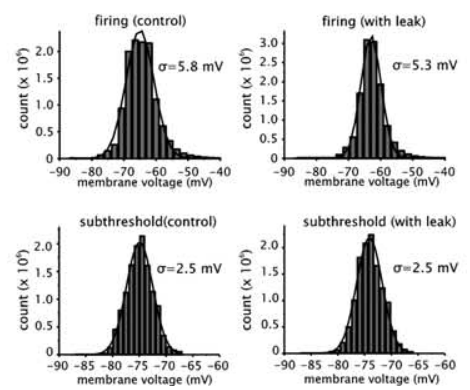

Figure 4. Conductance-based model reproduces effects of added leak conductance on spike frequency adaptation and ISI variability. $\boldsymbol{A}$, Voltage traces of model in response to step depolarizations to a steady-state firing frequency of $5 \mathrm{~Hz}$ under contro conditions (top) and with added leak conductance (bottom). $\boldsymbol{B}$, Plot of firing frequency as a function of time in model of adapting pyramidal cell under control conditions $(\bigcirc)$ and with added leak conductance $(\square)$. Lines denote a single-exponential fit to individual adaptation rates under each condition. C, Voltage traces of model driven with Gaussian current input under control conditions (top) and with added leak conductance (bottom). D, Histograms of ISI values under control conditions (top) and with added leak conductance (bottom). Bin size for histograms was $0.15 \mathrm{~s}$. E, Cumulative distribution plot of the ISI values generated in model under control conditions (gray) and with added leak conductance (black). $\boldsymbol{F}$, Histograms of voltage distributions from model in the subthreshold and spiking regime under control conditions or with added leak conductance. All distributions could be fit with a Gaussian function.

ian noise $(n=0.02)$ (Fig. $5 A)$. We were particularly interested in assessing the effect of added leak conductance on the fast subsystem of the model, before the adaptation variable has significantly changed and slowed spike firing. We considered this a reasonable reduction, because the time constant of adaptation is significantly slower than any other process in the model.

We started the analysis of the fast subsystem of the model by considering the voltage trajectory in response to a pulse of current of sufficient magnitude to elicit a single spike. This stimulus provides a direct method of assessing the amount of excitability after a single spike under control and with added leak conductance. As indicated in Figure $5 B$, a single pulse-elicited spike produces a substantially larger ADP under control conditions than with added leak conductance. We hypothesized that the decrease in the ADP with increased leak conductance could influence the variability of spike output by decreasing the excitability after a spike and reducing the number of high-frequency spike events. Because the fast subsystem is composed of only two differential equations, we can view the fast subsystem of the model in a phase-plane portrait under control and with added leak conductance (Fig. 5C). The voltage dynamics of the fast subsystem during the ADP can be understood if we examine the voltage nullcline with and without the extra leak during pulseelicited spiking. The voltage nullcline corresponds to a line in phase space in which the membrane voltage is constant $(d V /$ $d t=0$ ) (Fig. 5C). The closer the trajectory of the system gets to the voltage nullcline, the slower the voltage variable changes with respect to time. Likewise, the $h$ nullcline corresponds to a line in phase space where the $h$ variable is constant ( $d h$ / $d t=0$ ) (Fig. 5C). In addition to the nullclines, the phase-plane portrait also shows two fixed points. One of these points (blue circles) is a stable point (i.e., node) and corresponds to the resting membrane voltage of the fast subsystem ( $-68 \mathrm{mV}$ in this case). The second point (black squares) is an unstable point (i.e., saddle point). A closer look at the phase portrait of the fast subsystem of the model in the vicinity of the stable and unstable point reveals that the voltage nullcline changes shape and position with added leak conductance (Fig. 5C). Under control, the parabolic shape of the voltage nullcline (Fig. 5C, top) is shallower and is positioned closer to the $h$ nullcline than with the added leak conductance (Fig. 5C, bottom). Therefore, the slow change in voltage associated with the ADP is caused by the close proximity of the trajectory to the voltage nullcline as it approaches the stable point. As a result, the voltage trajectory associated with a single spike in response to a pulse of current under the control scenario has a significantly greater amount of 
depolarization after the spike than a comparable trajectory with added leak conductance (Fig. $5 B$ ). In terms of biophysics, this can be understood if we consider that a large ADP is generated by the approximate balancing of membrane currents, which leads to a small flow of net current and hence a slow change in membrane voltage. Given larger values for leak conductance, the balance in net current is disrupted and membrane voltage returns to rest more quickly, which results in a smaller ADP.

We followed by doing a bifurcation analysis of the fast subsystem of the model under control and with added leak conductance using driving current $\left(I_{E}\right)$ as the bifurcation parameter. A bifurcation consists of the creation or elimination of fixed points and limit cycles, which can often be associated with important transitions in a neuron (e.g., transition from rest to firing). A change in the bifurcation structure of the model can result in a significant change in the output characteristics of the model. Consequently, if adding leak conductance can change the bifurcation of the model, it can have a major impact on the output of the model (e.g., spike regularity). The analysis consists of tracking the fixed points of the model (or limit cycle) as a function of $I_{E}$. In both control and with added leak conductance, the fast subsystem of the model undergoes a saddlenode bifurcation of fixed points in the transition from rest to firing. As $I_{E}$ is increased, the stable node point (thin solid line corresponding to resting membrane voltage) and unstable saddle point (thin dashed line) coalesce, and, as a result, the new stable solution becomes the limit cycle (spike firing). Under control conditions, however, the fast subsystem has a small region of bistability (black dashed lines) in which the limit cycle (thick solid line, only the lower limit is shown) exists before the coalescing of the stable and unstable fixed points (Fig. 5D). Also important is the relative positions of the lower voltage limit of the spike (thick solid line) and spike threshold (asterisk). As indicated, the lower limit of the spike trajectory is above spike threshold under control conditions. Conversely, with added leak, the bistability is gone and spike threshold is now above the lower limit of the spike trajectory (Fig. 5E). The increased leak conductance alters the relative positions of spike threshold and the spike AHP minimum. Hence, similar to the phase-plane analysis of pulse-elicited spiking, the dynamics under steady-state firing suggest an increase in the amount of positive feedback after a spike. From a dynamical sys-
A

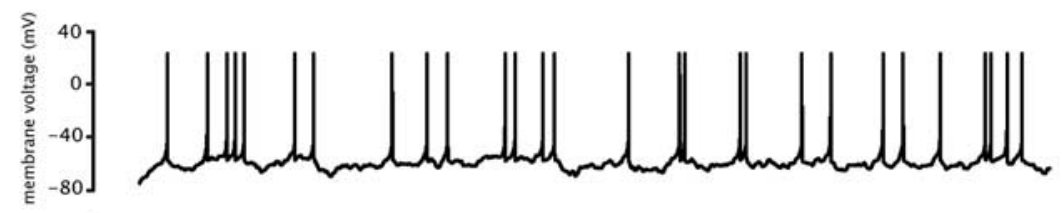

B

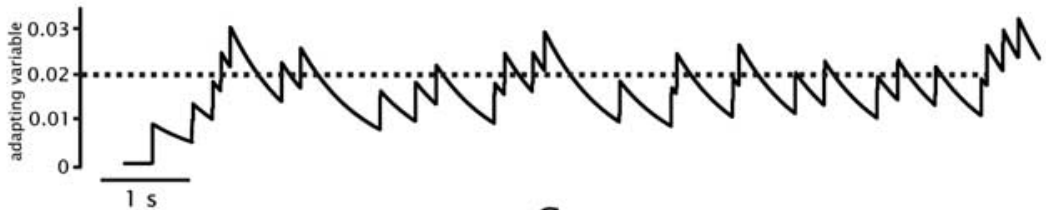

C
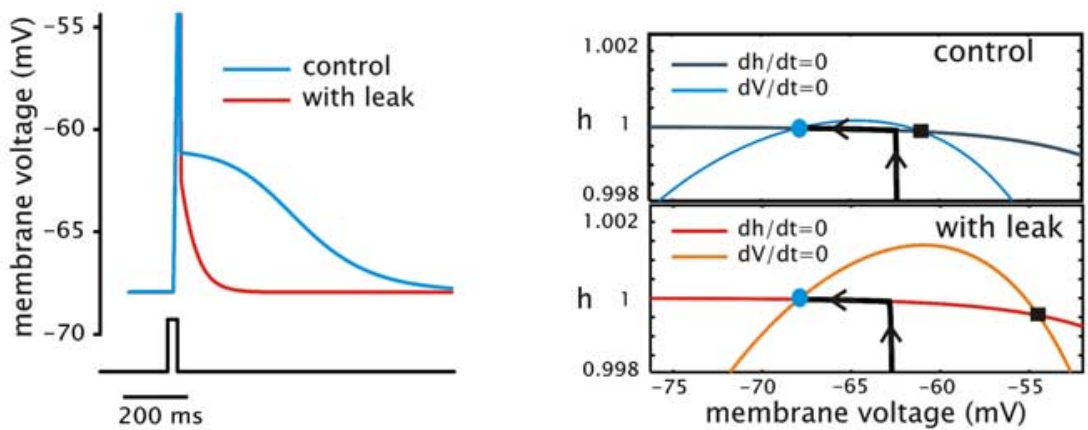

D

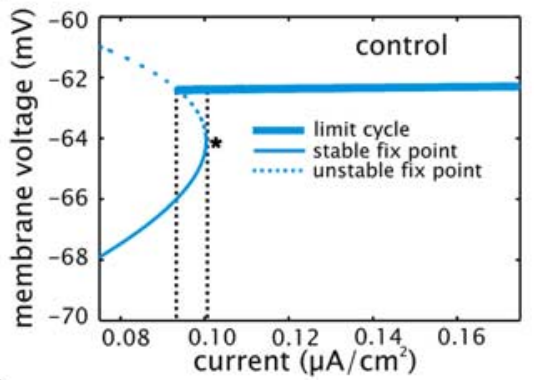

$\mathrm{F}$

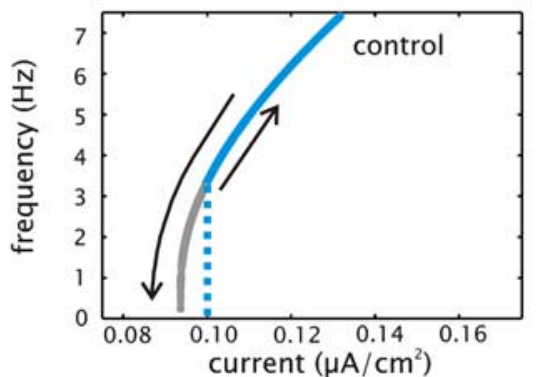

$\mathrm{E}$

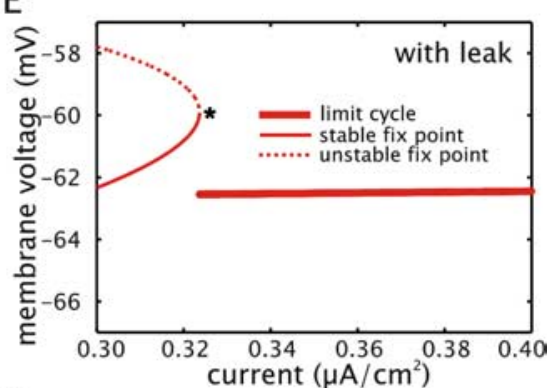

G

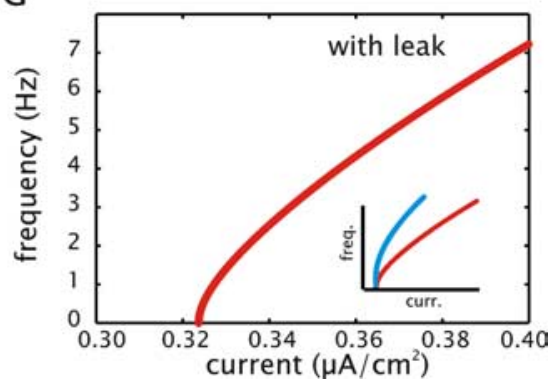

Figure 5. Analysis of the fast subsystem of the model can account for decrease in ISI variability and high-frequency spike events with increased leak conductance. $A$, Plot of the membrane voltage (top) and adaptation (bottom) variables in the three-equation model under control conditions. Model was driven with a Gaussian current input (SD of $0.13 \mu \mathrm{A} / \mathrm{cm}^{2}$ ) and a steady-state input current $\left(I_{E}\right)$ of $0.065 \mu \mathrm{A} / \mathrm{cm}^{2}$. B , Voltage trace of the fast subsystem of the model under control conditions (blue line) and with added leak conductance (red line). Model was held at a steady-state voltage of $-68 \mathrm{mV}$ using $0.065 \mu \mathrm{A} / \mathrm{cm}^{2}$ under control conditions and $0.1 \mu \mathrm{A} / \mathrm{cm}^{2}$ with increased leak conductance. A square pulse of current of $5 \mu \mathrm{A} / \mathrm{cm}^{2}$ magnitude and 10 ms duration was used to elicit a single spike under each condition. $C$, Phase-plane portraits of the fast subsystem of the model in the vicinity of the node and saddle point under control conditions (top) and with added leak conductance (bottom). Light blue and light red lines denote the voltage nullclines, whereas the $h$ nullclines are denoted with dark blue and dark red. Blue circles denote a stable node point (resting potential), and the black squares denotes the unstable saddle point. $\boldsymbol{D}, \boldsymbol{E}$, Bifurcation diagrams of the fast subsystem of the model under control conditions $(\boldsymbol{D})$ and with added leak conductance $(\boldsymbol{E})$. Thin solid lines and thin dashed lines denote the stable node and unstable saddle points, respectively. Thick solid lines denote the lower voltage limit of the limit cycle (spike). Asterisks denote the onset (spike threshold) of the limit cycle in the transition from rest to firing with increasing $I_{E}$. $\boldsymbol{F}, \boldsymbol{G}$, Plots of the $F$-/relationship of the fast subsystem of the model under control conditions $(\boldsymbol{F})$ and with added leak conductance $(\boldsymbol{G})$. The part of the $F-I$ curve shown with a gray line in $F$ denotes the region of bistability, which is associated with a discontinuity in the $F-/$ plot when the system is taken from rest to firing (black arrows). When the system is taken from firing to rest, however, the region of the $F$-l curve denoted by the gray line can be observed (an example of hysteresis). Inset in $G$ shows a comparison of the $F-I$ plots (blue line corresponds to control). For comparison, the plots were shifted so as to have a common origin. 
tems perspective, the model has undergone a change in how the limit cycle is terminated in the transition from firing to rest as $I_{E}$ is decreased (Izhikevich, 2007). Under control, the model undergoes a saddle homoclinic bifurcation, whereas with added leak conductance, the transition from firing to rest is mediated through a saddle-node on invariant circle bifurcation.

Previous work on dynamical systems has shown that the gain (slope) of the $F-I$ relationship of model neurons decreases as the limit cycle bifurcation transitions from a saddle homoclinic to a saddle-node on invariant circle (Izhikevich, 2007). Furthermore, this transition has been associated with biophysical parameters that decrease the level of depolarization in the post-spike period (Fernandez et al., 2007; Izhikevich, 2007). Thus, because the size of the ADP is reduced significantly with added leak conductance, there is a corresponding decrease in the gain of the $F-I$ relationship of the fast subsystem of the model (Fig. $5 F, G$ ). A high gain has been shown to result in a more variable spike train in leaky integrate-and-fire (LIF) models driven with noisy stimuli (Troyer and Miller, 1997). Therefore, the decrease in the CV of the model with added leak conductance can be explained as a consequence of a lower gain in the $F-I$ relationship resulting from the changes in the ADP and bifurcation in the fast subsystem of the model.

We also performed a bifurcation analysis of the full threeequation model. This analysis indicates an absence of bistability in the model (supplemental Fig. 2, available at www.jneurosci.org as supplemental material). The dynamics of the fast subsystem represent a snapshot of the three-equation model that does not apply once the adaptation variable is fully activated. Nevertheless, the fast subsystem can be considered relevant in trying to account for differences in high-frequency spike events in response to noisy stimulus in which the adaptation variable is expected to decrease transiently.

In summary, increased leak conductance significantly reduces the size of the ADP of the fast subsystem of the model. This effect is associated with a change in the limit cycle bifurcation, which is also associated with a change in the gain of the $F-I$ relationship. In particular, the reduction in gain provides a link between increased leak conductance and reduced ISI variability. By reducing the gain of the $F-I$ relationship of the first ISI, adding leak can decrease the number of high-frequency spike events in response to noisy stimuli and decrease the variability of spike output in the model. In particular, we would expect the gain in response to high-frequency fluctuations to be dampened by added leak.

\section{Increased leak conductance reduces the ADP and gain in adapting pyramidal cells}

A testable prediction arising from the analysis of the fast subsystem of the model is that the size of the ADP after a single spike in adapting pyramidal cells should be greater in control than with the added leak conductance. Moreover, this process should result in a substantially larger gain in the $F-I$ relationship in control compared with the added leak conductance. Specifically, the gain measured from the first ISI should be substantially reduced. The change in gain is an important and unique prediction arising from the analysis of the model because previous theoretical and experimental work has established that increased leak conductance at the soma does not normally change the gain of the $F-I$ relationship (Gabbiani et al., 1994; Holt and Koch, 1997; Doiron et al., 2001; Mitchell and Silver, 2003; Prescott and De Koninck, 2003; Mehaffey et al., 2005).

We first tested the response of pyramidal cells to pulses driving individual spikes under control and with added leak conduc- tance (Fig. $6 B$ ). For comparison, we have provided the results for the model under the same stimulus conditions (Fig. 6A). Pulse frequency was set to $10 \mathrm{~Hz}$ in both cases so that the effect of the adaptation current on the magnitude of the ADP could be observed. Under these conditions, we measured the magnitude of the ADP as the minimum voltage after the spike within the 100 ms window afforded by the $10 \mathrm{~Hz}$ stimulus.

Under control conditions, both the model and cell produced a large ADP after the first few ISIs that progressively decreased and eventually reached a steady-state voltage attributable to the adaptation process (Fig. $6 A, B$ ). In the experimental data, the difference between the depolarization values after the first and last spike was $4.1 \pm 0.7 \mathrm{mV}$ in control and $1.5 \pm 0.2$ with added leak $(p=0.01 ; n=6)$. Similarly, the model produces a difference of 4 and $1.6 \mathrm{mV}$ under control and with added leak conductance, respectively. We should note that we also measured the ability for added leak conductance to alter the post-spike period in nonadapting cells. Unlike the results in adapting cells, adding leak conductance had a relatively small effect on the size of the AHP in these cells (supplemental Fig. 3, available at www.jneurosci.org as supplemental material). Hence, whereas in adapting cells the integral of the ADP decreased from $0.70 \pm 0.13$ to $0.28 \pm 0.08$ $\mathrm{mV} \cdot \mathrm{s}(p=0.01 ; n=5)$ with added leak conductance, the AHP only changed from $-0.1 \pm 0.14$ to $0.01 \pm 0.07 \mathrm{mV} \cdot \mathrm{s}(p=0.3$; $n=5$ ) in non-adapting cells (supplemental Fig. 3, available at www.jneurosci.org as supplemental material).

We next measured the $F-I$ relationship and gain in adapting pyramidal cells. The $F-I$ relationship was measured at the beginning ("initial") and end of the spike train once spike frequency reached a steady value ("steady state"). As stated previously, the prediction from the fast subsystem of the model is that the gain measured from the initial ISI should be greater in control than with added leak, whereas the difference in gain at the end of the spike train should be reduced as a result of the adaptation process. The $F-I$ relationship was measured using square current pulses of $5 \mathrm{~s}$ duration in control or in the presence of $6 \mathrm{nS}$ of added leak conductance $\left(E_{\mathrm{rev}}=-70\right)$. Gain was calculated using a linear fit to the $F-I$ relationship $\left(r^{2}>0.95\right)$ (Fig. 6C,D).

In the three-equation model, leak reduces the initial gain from 91 to $48 \mathrm{~Hz} \cdot \mathrm{cm}^{2} / \mu \mathrm{A}$ and the steady-state gain from 26 to 17 $\mathrm{Hz} \cdot \mathrm{cm}^{2} / \mu \mathrm{A}$. A measurement of the initial and steady-state gain in the cell produced similar qualitative changes in gain between control and with the added leak conductance. The initial gain in the cell under control conditions was $201 \pm 22 \mathrm{~Hz} / \mathrm{nA}$, whereas with extra leak, the value decreased to $138 \pm 14 \mathrm{~Hz} / \mathrm{nA}$ ( $p=$ $0.003 ; n=8$ ) (Fig. $6 E$ ). Likewise, the steady-state gain in control was $87 \pm 11 \mathrm{~Hz} / \mathrm{nA}$, whereas with added leak, gain decreased to $67 \pm 12 \mathrm{~Hz} / \mathrm{nA}(p=0.004 ; n=8)$ (Fig. $6 E)$. The average decrease in the initial and steady-state gain caused by adding leak was $63 \pm$ 14 and $20 \pm 5 \mathrm{~Hz} / \mathrm{nA}$, respectively $(p=0.03 ; n=8$ ) (Fig. $6 F$ ). Although the changes in gain associated with adaptation were larger than those induced by leak on the initial gain, the initial change is important because it changed substantially with leak and is more relevant under noisy stimuli. These data confirmed the prediction of our model of a lower gain value with added leak conductance.

\section{Ability of leak to decrease the gain and $\mathrm{CV}$ of spike output depends on ADP dynamics}

To help substantiate our understanding of the dynamics of the cell, we considered experimental manipulations that could eliminate the ability for leak to reduce the $\mathrm{CV}$ and gain. A prediction arising from the model is that a decrease in the ADP (or a larger 
AHP under steady-state firing conditions) will diminish the ability for the added leak conductance to modulate the gain of the neuron. Essentially, a smaller ADP should convert the dynamics of adapting layer III cells to a more conventional behavior, whereby adding leak does not reduce the gain of the $F-I$ relationship nor the $\mathrm{CV}$ of the ISI distribution. In fact, a decrease in the ADP might also result in a higher CV with added leak conductance attributable to the decrease in $\tau_{m}$ and the corresponding decrease in integration time of the neuron. In the presence of a reduced ADP, adapting pyramidal cells should behave more similar to non-adapting cells with respect to increased leak conductance and its effect on ISI variability.

As before, an analysis of the fast subsystem of the model can explain how decreasing the ADP can alter the dynamics of the model such that adding leak fails to alter the CV and gain once the ADP is artificially reduced. As shown in Figure 7, an increase in the current underlying the AHP in the model ( $I_{\mathrm{KF}}$ was increased by 50 to $5.48 \mathrm{mS} / \mathrm{cm}^{2}$ ) eliminates the ability for added leak conductance to reduce the $\mathrm{CV}$ (Fig. $7 A, B$ ). A comparison of the ADP after a single spike reveals that, once the ADP is decreased, the ability for added leak conductance to change the ADP is reduced (compare Figs. 5B, 7C). Once again, an analysis of the fast subsystem of the model during the post-spike period can account for this observation. An increase in the current underlying the AHP reduces the time spent close to the voltage nullcline and hence the amount of depolarization after a spike (Fig. 7D). By reducing the amount of time spent close to the voltage nullcline, increasing $I_{\mathrm{KF}}$ reduces the potential influence that changes in the voltage nullcline brought on by increasing leak conductance can have on the voltage trajectory. A bifurcation analysis of the fast subsystem of the model indicates that increasing $I_{\mathrm{KF}}$ changes the relative positions of threshold and the lower voltage limit of the spike such that threshold is slightly above the lower voltage limit (Fig. 7E) and similar to the fast subsystem with added leak conductance (Fig. 5E). Furthermore, adding leak conductance no longer changes the relative positions of threshold and the lower voltage limit of the spike (Fig. $7 F$ ). A measurement of the F-I relationship of the fast subsystem shows that decreasing the ADP prevents an increase in leak conductance from decreasing the gain (Fig. 7G,H). Therefore, decreasing the ADP causes the same change in bifurcation that adding leak alone induced before.

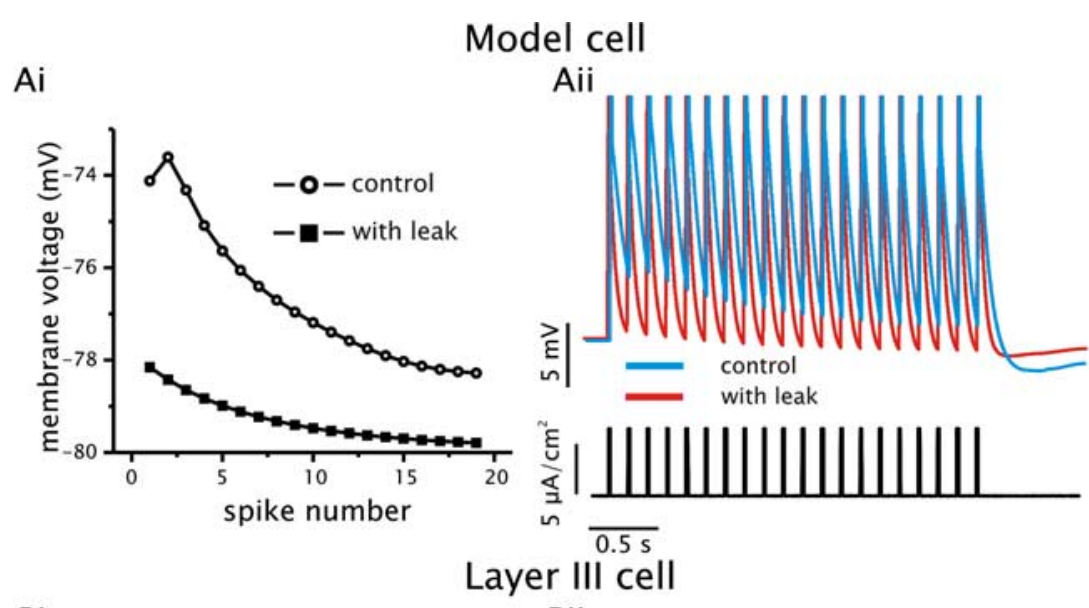

Bi

Bii
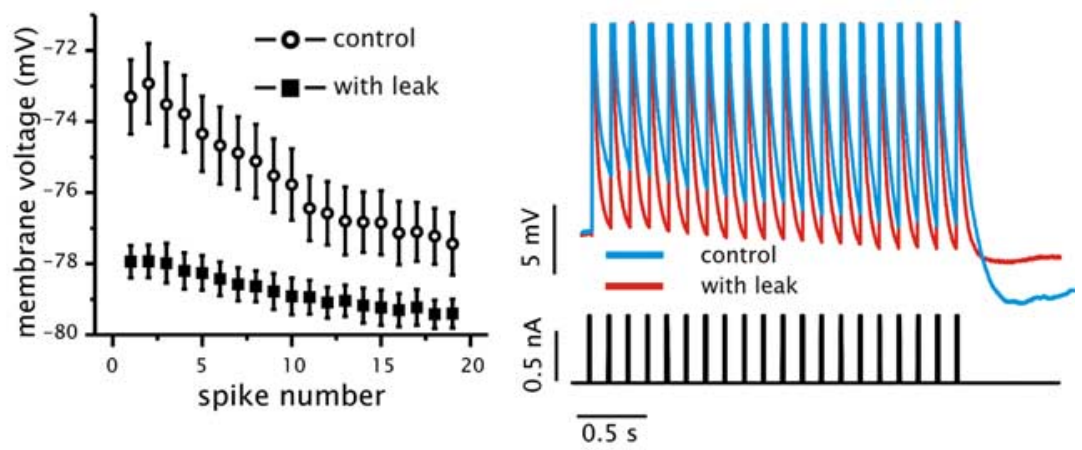

C

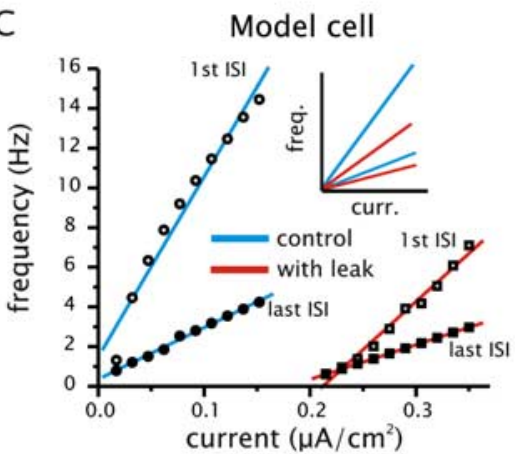

D
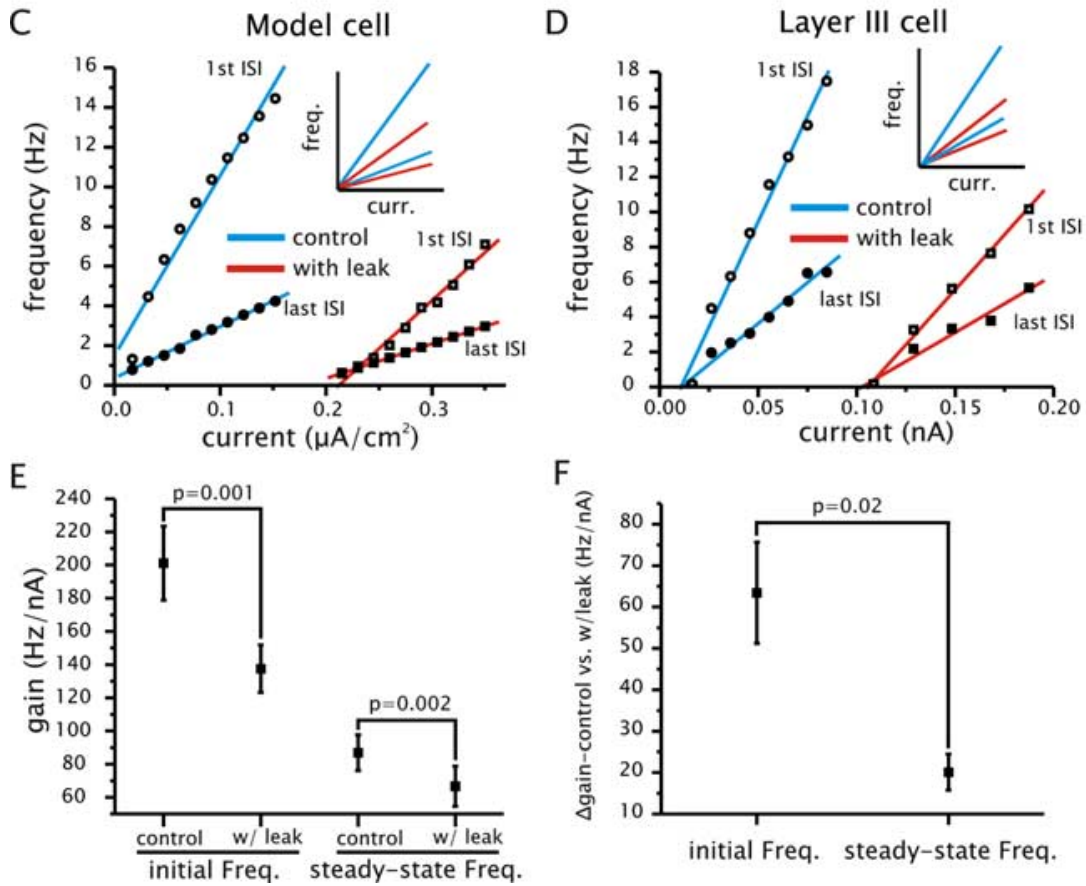

Figure 6. Increased leak conductance reduces the gain of the $F-/$ relationship in adapting pyramidal cells. $A, B$, Response of threeequation model $(\boldsymbol{A})$ and pyramidal cell $(\boldsymbol{B})$ to a $10 \mathrm{~Hz}$ stimulus eliciting single spikes. Model and cell were held at approximately $-80 \mathrm{mV}$ before delivery of $10 \mathrm{~Hz}$ stimulus (total of 20 pulses). The stimulus pulse ( $10 \mathrm{~ms}$ duration) had a magnitude $6 \mu \mathrm{A} / \mathrm{cm}^{2}$ in the model and 0.6 $\mathrm{nA}$ in the pyramidal cell. The magnitude of ADP elicited in response to the stimulus in the model ( $\boldsymbol{A} \boldsymbol{i})$ and cell $(\boldsymbol{B})$ was quantified and plotted as the voltage minimum between pulses (Aii, Bii). C, D, Initial and steady-state F-/ plots under control conditions (blue line) and with added leak conductance (red line) for the three-equation model ( $\boldsymbol{C}$ and pyramidal cell ( $\boldsymbol{D}$ ). Both model and cell were held at approximately $-80 \mathrm{mV}$ before delivery of square current steps of $5 \mathrm{~s}$ duration. Initial spike frequency was measured from the first ISI, whereas steadystate frequency was taken as the average of the last two ISI once firing frequency reached a steady-state value. In $C$ and $\boldsymbol{D}$, the insets show a comparison of the linear fits of the $F-I$ relationships. For comparison, lines were shifted so as to have a common origin. $\boldsymbol{E}$, Plot of the average initial and steady-state gain of the $F$-/ relationship of pyramidal cells under control conditions and with added leak conductance. $\boldsymbol{F}$, Plot of the average difference in gain between the control condition and with added leak conductance for the initial and steady-state $F-I$ relationships in pyramidal cells. 
$\mathrm{Ai}$

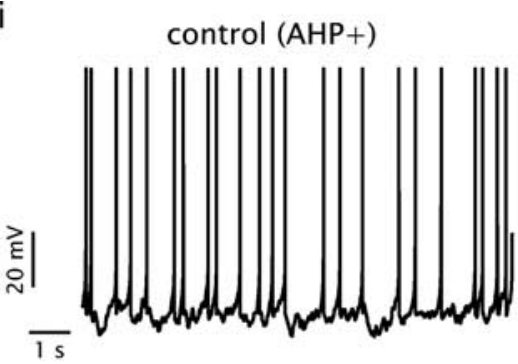

$\mathrm{Bi}$

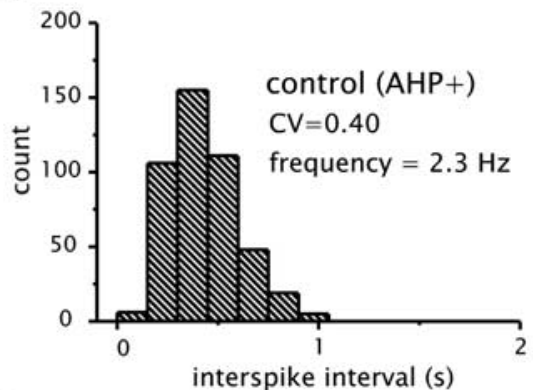

C

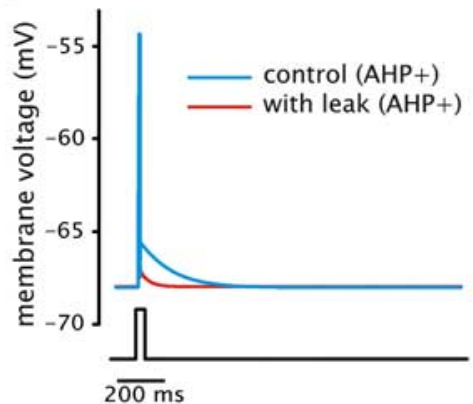

$\mathrm{E}$

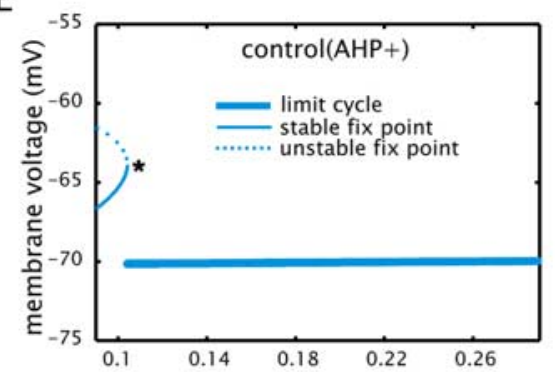

G

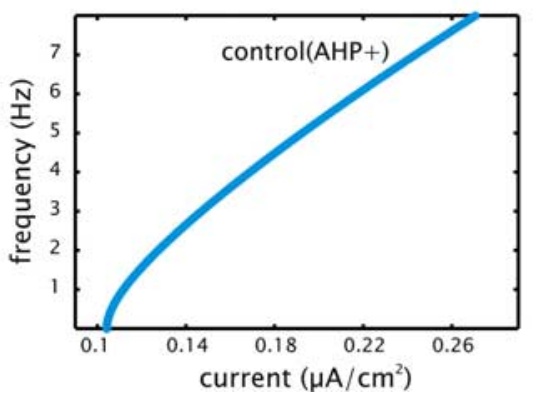

Aii

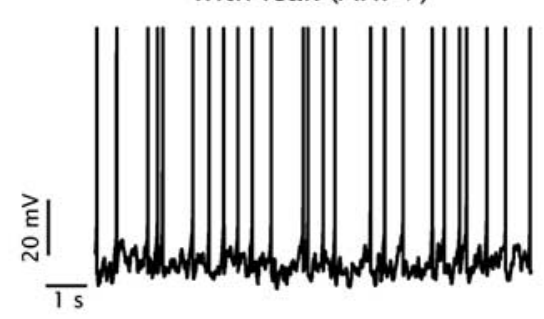

Bii

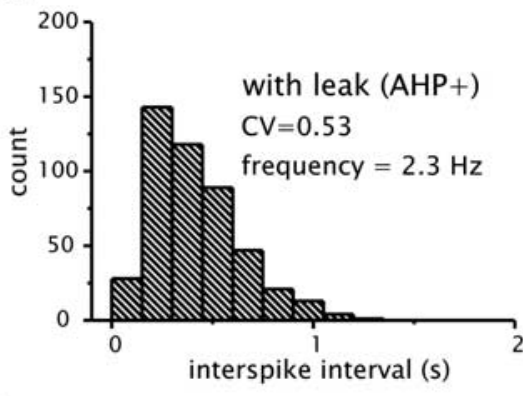

D

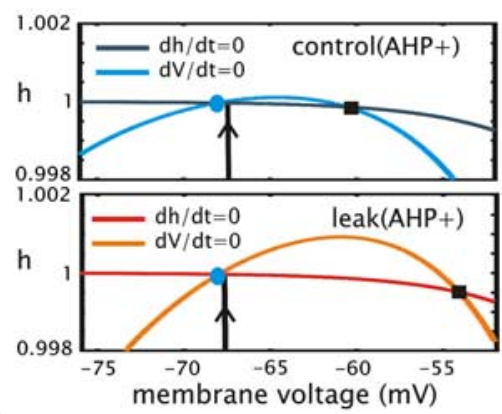

$\mathrm{F}$

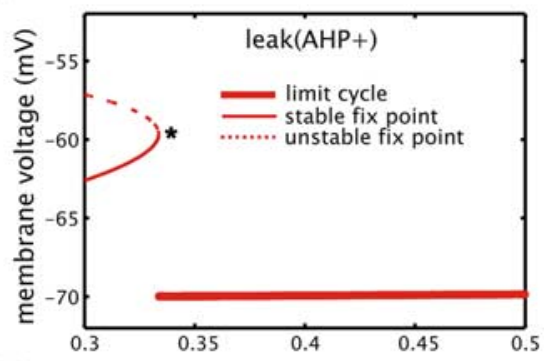

$\mathrm{H}$

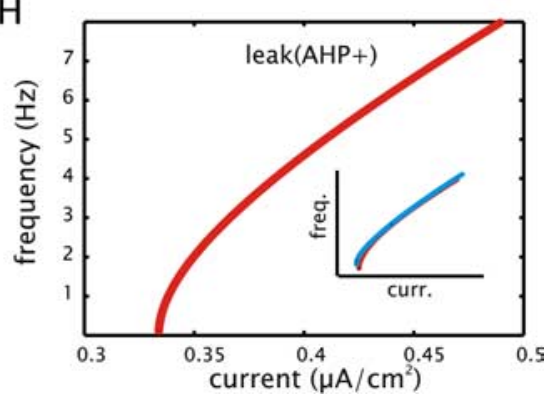

Figure 7. Increasing the size of the AHP in the three-equation model eliminates the ability for added leak conductance to increase spike output regularity. $\boldsymbol{A}$, Voltage traces of the model driven with a Gaussian current input under control conditions (Ai) and with added leak conductance $\left(\right.$ Aii) in the presence of increased repolarizing $\mathrm{K}^{+}$current $\left(I_{\mathrm{KF}} ; \mathrm{AHP}+\right)$. Histograms of ISI values under control conditions $(\boldsymbol{B} \boldsymbol{i})$ and with added leak conductance $(\boldsymbol{B} \boldsymbol{i})$ in the presence of increased $I_{\mathrm{KF}}$. Bin size was $0.15 \mathrm{~s}$. $\boldsymbol{C}$, Voltage trace of the fast subsystem of the model under control conditions (gray line) and with added leak conductance (black line) in the presence of increased $I_{\mathrm{KF}}$. Model was held at a steady-state voltage of $-68 \mathrm{mV}$ using $0.065 \mu \mathrm{A} / \mathrm{cm}^{2}$ under control conditions and $0.1 \mu \mathrm{A} / \mathrm{cm}^{2}$ with increased leak conductance. A square pulse of current of $5 \mu \mathrm{A} / \mathrm{cm}^{2}$ magnitude and $10 \mathrm{~ms}$ duration was used to elicit a single spike under each condition. $D$, Phase-plane portraits of the fast subsystem of the model in the vicinity of the node and saddle point under control conditions (top) and with added leak conductance (bottom) in the presence of increased $I_{\mathrm{KF}}$. Light blue

As a result, adding leak conductance after the ADP has been decreased no longer causes an additional change in the bifurcation structure and hence does not result in a decrease in the gain and CV of the ISI distribution. In biophysical terms, the increase in the hyperpolarizing current underlying the AHP disrupts the balance in conductances associated with a small net current flow and a large ADP. Once the post-spike period is dominated by the hyperpolarizing current, increasing leak conductance has a smaller effect on the size of ADP.

We followed by testing the prediction resulting from the analysis of the model. In essence, a reduction of the ADP of the cell should eliminate the ability for added leak conductance to decrease ISI variability. To decrease the ADP and increase the AHP in adapting pyramidal cells, we introduced a high-voltage-activated $\mathrm{K}^{+}$current $\left(I_{\mathrm{AHP}+}\right)$ using dynamic clamp (see Materials and Methods). In Figure 8, we show the ADP (Fig. 8A) and average AHP (Fig. $8 B$ ) under control and with added leak conductance in the presence (black line) or absence (gray line) of the $I_{\mathrm{AHP}+}$. Under these conditions, the difference in the initial gain of the $F-I$ relationship between control and with added leak conductance was only $14 \pm 2 \mathrm{~Hz} / \mathrm{nA}(n=7)$. As before, we measured the CV from $200 \mathrm{~s}$ sweeps in cells stimulated with Gaussian noise under control or with $6 \mathrm{nS}$ of added leak conductance (firing at $\sim 2 \mathrm{~Hz}$ ). Under control conditions, without the modified AHP, the average $\mathrm{CV}$ was $0.78 \pm 0.05$, whereas with added leak, this value decreased to $0.63 \pm 0.03(p=0.007 ; n=6)$ (Fig. $8 C-$ $E)$. Under the same stimulus conditions and set of cells but now with the modified AHP, the CV under control was $0.52 \pm$ 0.03 , whereas with added leak, the value was $0.58 \pm 0.03(p=0.1 ; n=6)$ (Fig.

and light red lines denote the voltage nullclines, and the $h$ nullclines are denoted with dark blue and dark red. Blue circles denote a stable node point (resting potential), and the black squares denotes the unstable saddle point. $\boldsymbol{E}, \boldsymbol{F}$, Bifurcation diagrams of the fast subsystem of the model under control conditions $(\boldsymbol{E})$ and with added leak conductance $(\boldsymbol{F})$ in the presence of increased $I_{\mathrm{KF}}$. Thin solid lines and thin dashed lines denote the stable node and unstable saddle points, respectively. Thick solid lines denotes the lower voltage limit of the limit cycle (spike). Asterisks denote the onset (spike threshold) of the limit cycle in the transition from rest to firing with increasing $I_{E}, \boldsymbol{G}, \boldsymbol{H}$, Plots of the $F-$ / relationship of the fast subsystem of the model under control conditions $(\boldsymbol{G})$ and with added leak conductance $(\boldsymbol{H})$ in the presence of increased $I_{K F}$. Inset in $\boldsymbol{H}$ shows a comparison of the $F-I$ plots (blue line corresponds to control). For comparison, the plots were shifted so as to have a common origin. 
$8 C-E)$. Consequently, the average change in the $\mathrm{CV}$ induced by adding leak was $-0.15 \pm 0.04$ without the modified AHP and $0.06 \pm 0.04$ with the modified AHP $(p=0.003 ; n=6)$ (Fig. $8 F)$. These data support the hypothesis that the interaction between the ADP dynamics and leak conductance are critical in determining ISI variability.

\section{Discussion}

Using dynamic-clamp experiments and conductance-based modeling, we have shown that the capacity of increased leak conductance to alter ISI variability depends on the neuron type. In the examples provided in our study, the ability to increase the variability of the spike train through a high conductance state hinges on the ADP dynamics present in the neuron. As a result, a synaptic conductance stimulus can decrease the CV of spike output relative to a comparable synaptic current stimulus.

Previous work in vitro has shown that Poisson distributed conductance-based synapses can only account for a portion of the high ISI variability observed in vivo (Harsch and Robinson, 2000). Additional work has suggested that accounting for a greater portion of ISI variability observed in vivo requires taking into account more complex forms of statistics in the synaptic input delivered to neurons, such as correlations in the stimulus (Stevens and Zador, 1998; Harsch and Robinson, 2000; DeWeese and Zador, 2006). Before this study, the ability for a Poisson-distributed conductance-based input to increase the ISI variability above that of a comparable current-based input, however, had not been tested experimentally. As a result, it was unclear whether the high conductance state by itself contributed to an increase in ISI variability in neurons, as suggested by the theoretical and modeling work (Stein, 1965; Bernander et al., 1991; Koch, 1999; Moreno-Bote and Parga, 2005). By systematically comparing ISI variability under comparable current- and conductancebased stimuli in two different cell types, we have shown that increased conductance can contribute to either an increase or decrease in ISI variability.

Decrease in ISI variability is caused by a reduction in gain and high-frequency spike events

The decrease in ISI variability in adapting pyramidal cells is a direct result of a lower gain with increased leak conductance. A previous study of cortical neurons using an LIF model has linked gain, AHP reset, and ISI variability (Troyer and Miller, 1997). By providing a more positive reset value for the AHP in the LIF model, the authors found that ISI variability could be signifi-
$\mathrm{Ai}$
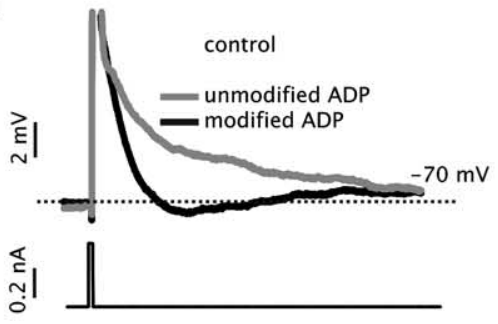

$\overline{100 \mathrm{~ms}}$

$\mathrm{Bi}$

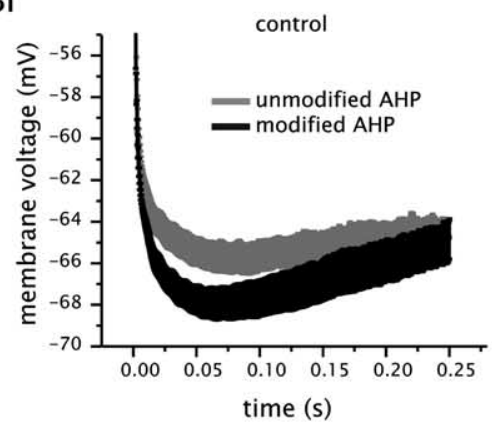

$\mathrm{Ci}$

Ciii

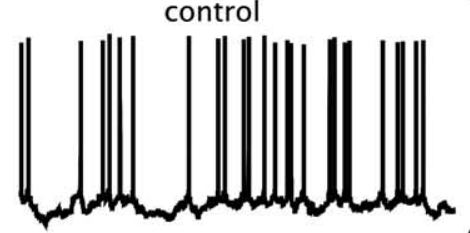

control (AHP+)

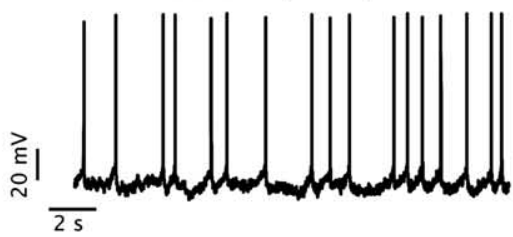

$D$
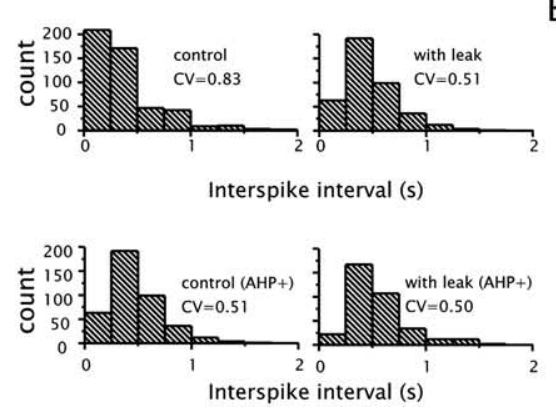

E

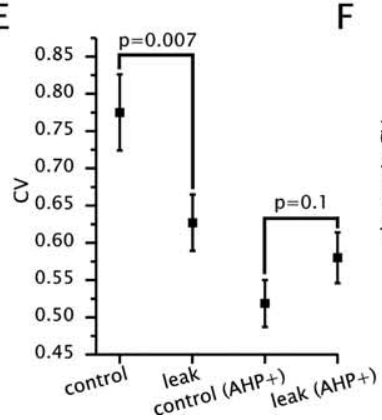

Aii

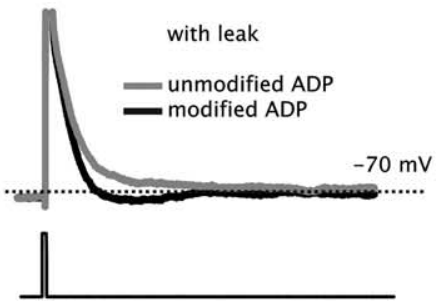

Bii

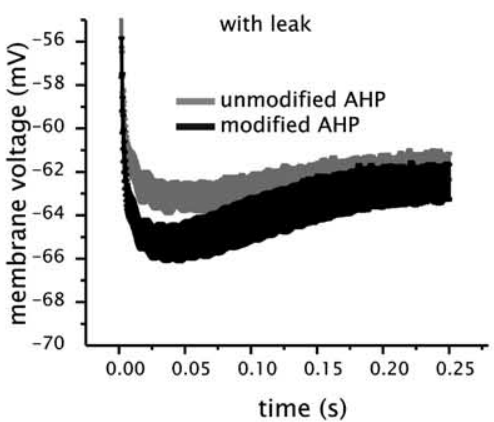

Cii
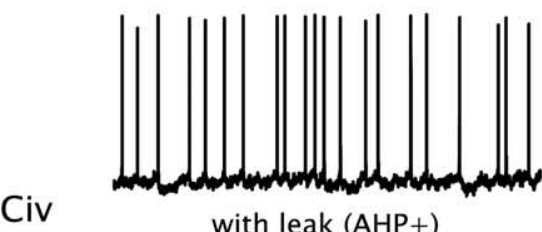

with leak (AHP+)

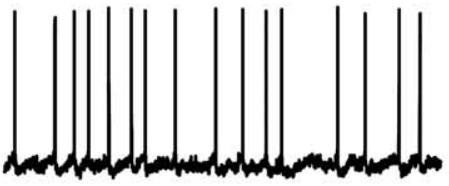

Figure 8. Increasing the size of the AHP in adapting layer III pyramidal cells eliminates the ability for added leak conductance to increase ISI variability. $\boldsymbol{A}$, Comparison of ADP under control conditions (Ai) and with added leak conductance (Aii) in the presence (black line) or absence (gray line) of the AHP modifying current. $\boldsymbol{B}$, Average AHP under control conditions ( $\boldsymbol{B} \boldsymbol{i}$ ) and with added leak conductance ( $\mathbf{B i i}$ ) in the presence (black line) or absence (gray line) of the AHP modifying current. For each cell, the AHP was taken as the average during the $200 \mathrm{~s}$ sweeps used to calculate the $\mathrm{CV}$ for a period of $0.25 \mathrm{~s}$ after spike threshold. Line widths denote extent of error bars. $\boldsymbol{C}$, Examples of voltage traces from a single cell in control (Ci, $\boldsymbol{C i i i )}$ and with added leak conductance (Cii, Civ) in the presence (Ciii, Civ) or absence (Ci, Cii) of the AHP modifying current. D, Examples of histograms of ISI values from a single cell under conditions described in $\boldsymbol{C}$. Bin size for histograms was $0.2 \mathrm{~s}$. $\boldsymbol{E}$, Plot of average $\mathrm{CV}$ under conditions described in C. F, Plot of average change in CV induced by adding leak conductance in the absence or presence of the increased AHP.

cantly increased over previous models, which provided a closer match to experimental results (Troyer and Miller, 1997). Our data and model support these conclusions, although the dynamics underlying the AHP can be modified with increased leak con- 
ductance. Consequently, the initial gain of the model and neuron, before adaptation has reached its maximum magnitude, is significantly reduced with added leak conductance.

Previous experiments have failed to demonstrate a change in gain with different levels of steady-state leak conductance introduced via dynamic clamp (Chance et al., 2002; Mitchell and Silver, 2003; Prescott and De Koninck, 2003). Our model and data suggest that the ability of leak to alter gain will depend on the specific dynamics present in the neuron. Accordingly, within the vicinity of the transition between the two types of limit cycle bifurcations (homoclinic vs invariant cycle), leak can alter the gain of the neuron. Once the neuron is far from this transition point, as happens when the AHP is increased artificially, increasing leak fails to reduce the gain and decrease the $\mathrm{CV}$.

\section{ADP dynamics can be significantly influenced by increased leak conductance}

The analyses of the adapting pyramidal cell model and experimental data show that increased leak conductance can significantly change the ADP. Under control levels of leak conductance, the fast subsystem of the model generates a large ADP, bistability, and is associated with a high gain in the $F-I$ relationship. The large ADP and bistability are the result of weak repolarizing dynamics $\left(I_{\mathrm{KS}}\right.$ and $I_{\mathrm{Na}}$ inactivation) and a large $\tau_{m}$, which permit a significant amount of depolarization to follow a single spike. In models with true threshold behavior and incorporating a reset mechanism to simulate the AHP, bistability can be introduced by setting the reset voltage after a spike to a value more positive than threshold (Izhikevich, 2003). The generation of a spike leads to persistent activity. If the spike reset is set below threshold, however, the bistability is eliminated. By decreasing the magnitude of the ADP, added leak conductance eliminates bistability in the fast subsystem in a manner analogous to using a more negative voltage reset.

Modeling work in cerebellar Purkinje cells has shown that increased leak conductance can eliminate bistability by reducing the ADP (Fernandez et al., 2007). Similar to the fast timescale of adapting pyramidal cells, Purkinje cells have been found to have a large ADP caused, in part, by a large $\tau_{m}$ (Fernandez et al., 2007). Furthermore, bistability in Purkinje cells has been shown to be caused by a saddle homoclinic bifurcation (Fernandez et al., 2007), similar to the fast subsystem of the adapting pyramidal cell model under control conditions.

Although our analysis indicates a degree of bistability within the fast timescale of adapting pyramidal cells, neither the model nor the cell expressed bimodality in the membrane voltage or ISI distributions. In the model, the lack of any characteristic signatures of bistability is a result of two factors. First, the bistability in the fast subsystem of a model occurs over a small region of $I_{E}$, and hence is relatively weak. Second, the $\mathrm{K}^{+}$current responsible for adaptation $\left(I_{\mathrm{KS}}\right)$ decreases the post-spike depolarization within a few spikes, eliminating bistability at timescales longer than the first ISI. Thus, the bistable behavior present in the fast subsystem is relevant only within brief epochs of activity. This said, the model and data suggest that a small region of bistability in the fast subsystem can have a significant impact on the number of highfrequency spike events and degree of spike output regularity through the associated consequences on the ADP and gain. A previous study has shown experimental evidence consistent with a degree of bistability in layer III pyramidal cells. In a study by Yoshida and Alonso (2007), in which the ADP was increased pharmacologically, it was found that a single brief current pulse could elicit a prolonged depolarization that produced spiking over several seconds. These results are consistent with the pres- ence of weak bistable dynamics that can be expressed more robustly once the ADP is enhanced.

The ADP of non-adapting cells was not significantly changed by the addition of a steady-state leak conductance (supplemental Fig. 3, available at www.jneurosci.org as supplemental material). In non-adapting cells, the post-spike period often consisted of a brief hyperpolarization, with the average integral of the membrane voltage being negative. This suggests that, in non-adapting cells, hyperpolarizing currents are more dominant in the postspike period than in adapting cells. The ability for added leak to modify the post-spike voltage depends on the degree to which any single conductance dominates. Under conditions in which, for example, a $\mathrm{K}^{+}$conductance is the only major contributor to membrane conductance, increased leak conductance will have a small effect. However, under conditions in which membrane conductances are balanced and net current flow is near zero, such as during a prominent ADP or plateau potential, adding leak can have a significant effect on the membrane voltage trajectory by disrupting the balance in net current. Hence, once the ADP was artificially decreased with dynamic clamp, adding leak had a minor effect on the ADP magnitude (Fig. $8 \mathrm{~A}$ ).

Previous work in hippocampal pyramidal cells, as well other cell types, has shown that the ADP associated with pulse-elicited spike generation can contribute to burst generation (Thompson and Smith, 1976; Lemon and Turner, 2000; Metz et al., 2005; Yue et al., 2005). A decrease in the amplitude of the ADP induced through pharmacological agents have been associated with significant reductions in the bursting behavior of neurons (Metz et al., 2005; Yue et al., 2005). This line of work has tended to emphasize the ability of voltage-gated conductances to sustain the ADP. In contrast, our work shows that the amount of leak conductance can also play a significant role in the size of the ADP. As a result, the amount of leak conductance associated with background synaptic activity may exert an influence on the likelihood of generating burst events.

\section{Implications for entorhinal cortical activity in vivo}

Experimental work has shown that, in the presence of a muscarinic receptor agonist, lateral entorhinal cortical (LEC) layer III pyramidal cells are capable of producing persistent activity in a form similar to the activity noted previously in cerebellar Purkinje cells (Tahvildari et al., 2007, 2008). A large ADP mediated by a nonspecific cation current is believed to underlie persistent activity in LEC pyramidal cells. A more complex form of graded persistent activity, also evoked with muscarinic receptor activation and mediated by an ADP, has been reported in layer V MEC pyramidal cells (Egorov et al., 2002; Fransen et al., 2006). Our results suggest that these types of dynamics could be susceptible to changes in membrane conductance provided that a fine balance in membrane conductances is a contributing factor to the voltage trajectory of the ADP. However, if any single conductance dominates the generation of the ADP, the effects of leak will be less. Consequently, it is possible that certain types of bistability and graded persistent activity are abolished under more realistic in vivo levels of activity.

\section{References}

Alonso A, Llinás RR (1989) Subthreshold $\mathrm{Na}^{+}$-dependent theta-like rhythmicity in stellate cells of entorhinal cortex layer II. Nature 342:175-177.

Anderson JS, Lampl I, Gillespie DC, Ferster D (2000) The contribution of noise to contrast invariance of orientation tuning in cat visual cortex. Science 290:1968-1972.

Berg RW, Alaburda A, Hounsgaard J (2007) Balanced inhibition and excitation drive spike activity in spinal half-centers. Science 315:390-393. 
Bernander O, Douglas RJ, Martin KA, Koch C (1991) Synaptic background activity influences spatiotemporal integration in single pyramidal cells. Proc Natl Acad Sci U S A 88:11569-11573.

Bettencourt JC, Lillis KP, Stupin LR, White JA (2008) Effects of imperfect dynamic clamp: computational and experimental results. J Neurosci Methods 169:282-289.

Borg-Graham LJ, Monier C, Frégnac Y (1998) Visual input evokes transient and strong shunting inhibition in visual cortical neurons. Nature 393:369-373.

Cardin JA, Palmer LA, Contreras D (2008) Cellular mechanisms underlying stimulus-dependent gain modulation in primary visual cortex neurons in vivo. Neuron 59:150-160.

Chance FS, Abbott LF, Reyes AD (2002) Gain modulation from background synaptic input. Neuron 35:773-782.

Destexhe A, Rudolph M, Paré D (2003) The high-conductance state of neocortical neurons in vivo. Nat Rev Neurosci 4:739-751.

DeWeese MR, Zador AM (2006) Non-Gaussian membrane potential dynamics imply sparse, synchronous activity in auditory cortex. J Neurosci 26:12206-12218.

Dickson CT, Mena AR, Alonso A (1997) Electroresponsiveness of medial entorhinal cortex layer III neurons in vitro. Neuroscience 81:937-950.

Doiron B, Longtin A, Berman N, Maler L (2001) Subtractive and divisive inhibition: effect of voltage-dependent inhibitory conductances and noise. Neural Comput 13:227-248.

Dorval AD, Christini DJ, White JA (2001) Real-Time linux dynamic clamp: a fast and flexible way to construct virtual ion channels in living cells. Ann Biomed Eng 29:897-907.

Egorov AV, Hamam BN, Fransén E, Hasselmo ME, Alonso AA (2002) Graded persistent activity in entorhinal cortex neurons. Nature 420:173-178.

Erchova I, Kreck G, Heinemann U, Herz AV (2004) Dynamics of rat entorhinal cortex layer II and III cells: characteristics of membrane potential resonance at rest predict oscillation properties near threshold. J Physiol 560:89-110.

Ermentrout B (1998) Linearization of F-I curves by adaptation. Neural Comput 10:1721-1729.

Ermentrout B (2002) Simulating, analyzing, and animating dynamical systems: a guide to XPPAUT for researchers and students. Philadelphia: Society for Industrial and Applied Mathematics.

Fernandez FR, White JA (2008) Artificial synaptic conductances reduce subthreshold oscillations and periodic firing in stellate cells of the entorhinal cortex. J Neurosci 28:3790-3803.

Fernandez FR, Engbers JD, Turner RW (2007) Firing dynamics of cerebellar Purkinje cells. J Neurophysiol 98:278-294.

Frank LM, Brown EN, Wilson MA (2001) A comparison of the firing properties of putative excitatory and inhibitory neurons from CA1 and the entorhinal cortex. J Neurophysiol 86:2029-2040.

Fransén E, Tahvildari B, Egorov AV, Hasselmo ME, Alonso AA (2006) Mechanism of graded persistent cellular activity of entorhinal cortex layer v neurons. Neuron 49:735-746.

Gabbiani F, Midtgaard J, Knöpfel T (1994) Synaptic integration in a model of cerebellar granule cells. J Neurophysiol 72:999-1009.

Giocomo LM, Zilli EA, Fransén E, Hasselmo ME (2007) Temporal frequency of subthreshold oscillations scales with entorhinal grid cell field spacing. Science 315:1719-1722.

Gloveli T, Schmitz D, Empson RM, Heinemann U (1997a) Frequencydependent information flow from the entorhinal cortex to the hippocampus. J Neurophysiol 78:3444-3449.

Gloveli T, Schmitz D, Empson RM, Dugladze T, Heinemann U (1997b) Morphological and electrophysiological characterization of layer III cells of the medial entorhinal cortex of the rat. Neuroscience 77:629-648.

Greenhill SD, Jones RS (2007) Simultaneous estimation of global background synaptic inhibition and excitation from membrane potential fluctuations in layer III neurons of the rat entorhinal cortex in vitro. Neuroscience 147:884-892.

Harsch A, Robinson HP (2000) Postsynaptic variability of firing in rat cortical neurons: the roles of input synchronization and synaptic NMDA receptor conductance. J Neurosci 20:6181-6192.

Holt GR, Koch C (1997) Shunting inhibition does not have a divisive effect on firing rates. Neural Comput 9:1001-1013.

Izhikevich EM (2003) Simple model of spiking neurons. IEEE Trans Neural Netw 14:1569-1572.
Izhikevich EM (2007) Dynamical systems in neuroscience: the geometry of excitability and bursting. Cambridge, MA: MIT.

Jörntell H, Ekerot CF (2006) Properties of somatosensory synaptic integration in cerebellar granule cells in vivo. J Neurosci 26:11786-11797.

Klink R, Alonso A (1993) Ionic mechanisms for the subthreshold oscillations and differential electroresponsiveness of medial entorhinal cortex layer II neurons. J Neurophysiol 70:144-157.

Klink R, Alonso A (1997) Morphological characteristics of layer II projection neurons in the rat medial entorhinal cortex. Hippocampus 7:571-583.

Koch C (1999) Biophysics of computation: information processing in single neurons. New York: Oxford UP.

Lemon N, Turner RW (2000) Conditional spike backpropagation generates burst discharge in a sensory neuron. J Neurophysiol 84:1519-1530.

Longtin A (2000) Effect of noise on the tuning properties of excitable systems. Chaos Solitons Fractals 11:1835-1848.

Mariño J, Schummers J, Lyon DC, Schwabe L, Beck O, Wiesing P, Obermayer K, Sur M (2005) Invariant computations in local cortical networks with balanced excitation and inhibition. Nat Neurosci 8:194-201.

Mehaffey WH, Doiron B, Maler L, Turner RW (2005) Deterministic multiplicative gain control with active dendrites. J Neurosci 25:9968-9977.

Metz AE, Jarsky T, Martina M, Spruston N (2005) R-type calcium channels contribute to afterdepolarization and bursting in hippocampal CA1 pyramidal neurons. J Neurosci 25:5763-5773.

Miller KD, Troyer TW (2002) Neural noise can explain expansive, powerlaw nonlinearities in neural response functions. J Neurophysiol 87:653-659.

Mitchell SJ, Silver RA (2003) Shunting inhibition modulates neuronal gain during synaptic excitation. Neuron 38:433-445.

Moreno-Bote R, Parga N (2005) Membrane potential and response properties of populations of cortical neurons in the high conductance state. Phys Rev Lett 94:088103.

Prescott SA, De Koninck Y (2003) Gain control of firing rate by shunting inhibition: roles of synaptic noise and dendritic saturation. Proc Natl Acad Sci U S A 100:2076-2081.

Prescott SA, Ratté S, De Koninck Y, Sejnowski TJ (2006) Nonlinear interaction between shunting and adaptation controls a switch between integration and coincidence detection in pyramidal neurons. J Neurosci 26:9084-9097.

Rudolph M, Pospischil M, Timofeev I, Destexhe A (2007) Inhibition determines membrane potential dynamics and controls action potential generation in awake and sleeping cat cortex. J Neurosci 27:5280-5290.

Shadlen MN, Newsome WT (1998) The variable discharge of cortical neurons: implications for connectivity, computation, and information coding. J Neurosci 18:3870-3896.

Softky WR, Koch C (1993) The highly irregular firing of cortical cells is inconsistent with temporal integration of random EPSPs. J Neurosci 13:334-350.

Stein RB (1965) A theoretical analysis of neuronal variability. Biophys J 5:173-194.

Steriade M, Timofeev I, Grenier F (2001) Natural waking and sleep states: a view from inside neocortical neurons. J Neurophysiol 85:1969-1985.

Stevens CF, Zador AM (1998) Input synchrony and the irregular firing of cortical neurons. Nat Neurosci 1:210-217.

Tahvildari B, Fransén E, Alonso AA, Hasselmo ME (2007) Switching between "On" and "Off" states of persistent activity in lateral entorhinal layer III neurons. Hippocampus 17:257-263.

Tahvildari B, Alonso AA, Bourque CW (2008) Ionic basis of ON and OFF persistent activity in layer III lateral entorhinal cortical principal neurons. J Neurophysiol 99:2006-2011.

Thompson SH, Smith SJ (1976) Depolarizing afterpotentials and burst production in molluscan pacemaker neurons. J Neurophysiol 39:153-161.

Troyer TW, Miller KD (1997) Physiological gain leads to high ISI variability in a simple model of a cortical regular spiking cell. Neural Comput 9:971-983.

Wolfart J, Debay D, Le Masson G, Destexhe A, Bal T (2005) Synaptic background activity controls spike transfer from thalamus to cortex. Nat Neurosci 8:1760-1767.

Yoshida M, Alonso A (2007) Cell-type specific modulation of intrinsic firing properties and subthreshold membrane oscillations by the $\mathrm{M}(\mathrm{Kv} 7)$-current in neurons of the entorhinal cortex. J Neurophysiol 98:2779-2794.

Yue C, Remy S, Su H, Beck H, Yaari Y (2005) Proximal persistent $\mathrm{Na}^{+}$ channels drive spike afterdepolarizations and associated bursting in adult CA1 pyramidal cells. J Neurosci 25:9704-9720. 